\title{
Adaptive Robust Motion Control of Direct-Drive DC Motors with Continuous Friction Compensation
}

\author{
Jianyong Yao, ${ }^{1}$ Guichao Yang, ${ }^{1}$ Zongxia Jiao, ${ }^{2}$ and Dawei $\mathrm{Ma}^{1}$ \\ ${ }^{1}$ The School of Mechanical Engineering, Nanjing University of Science and Technology, Nanjing 210094, China \\ ${ }^{2}$ The School of Automation Science and Electrical Engineering, Beihang University, Beijing 100191, China \\ Correspondence should be addressed to Jianyong Yao; jerryyao.buaa@gmail.com
}

Received 4 September 2013; Accepted 12 September 2013

Academic Editor: Carlo Bianca

Copyright (c) 2013 Jianyong Yao et al. This is an open access article distributed under the Creative Commons Attribution License, which permits unrestricted use, distribution, and reproduction in any medium, provided the original work is properly cited.

\begin{abstract}
Uncertainties including the structured and unstructured, especially the nonlinear frictions, always exist in physical servo systems and degrade their tracking accuracy. In this paper, a practical method named adaptive robust controller (ARC) is synthesized with a continuous differentiable friction model for high accuracy motion control of a direct-drive dc motor, which results in a continuous control input and thus is more suitable for application. To further reduce the noise sensitivity and improve the tracking accuracy, a desired compensation version of the proposed adaptive robust controller is also developed and its stability is guaranteed by a proper robust law. The proposed controllers not only account for the structured uncertainties (e.g., parametric uncertainties) but also for the unstructured uncertainties (e.g., unconsidered nonlinear frictions). Furthermore, the controllers theoretically guarantee a prescribed output tracking transient performance and final tracking accuracy in both structured and unstructured uncertainties while achieving asymptotic output tracking in the absence of unstructured uncertainties, which is very important for high accuracy control of motion systems. Extensive comparative experimental results are obtained to verify the high-performance nature of the proposed control strategies.
\end{abstract}

\section{Introduction}

Recently, there are many requirements for high-performance tracking control in industries [1-3], like robots [4], gantry systems [5], active suspension systems [6], micromechanisms [7], motion platforms [8], and so on. In these applications, direct-drive dc motor is widely employed since it is free from backlash and easy to realize for precise, high-speed, and highacceleration motion. However, designing high-performance tracking controllers for direct-driven motion systems are still not easy since designers are likely to encounter lots of nonlinearities and modeling uncertainties, including structured uncertainties (e.g., unknown payloads [9]) and unstructured uncertainties (e.g., unmodeled nonlinear frictions [10], cutting forces, unmodeled dynamics [11], and so on). These nonlinearities and uncertainties could severely deteriorate the achievable control performance, leading to undesirable control accuracy, limit cycles, and even instability [12]. Therefore, more effective approaches to achieve higher motion precision against nonlinearities and uncertainties are still pursued in industrial applications.
For the known nonlinearities existing in electrical drive systems, model-based controllers (e.g., feedback linearization control) are generally suitable for high-performance control purpose. But no matter how accurate the mathematical models of dynamic nonlinearities and parameter identification are, there always exist many hard-to-model terms in physical control systems. A typical hard-to-model example is the friction, and developing an accurate model for friction has been historically challenging [13].

When the nonlinearity can be linearly parameterized, adaptive control is useful in controlling this type of system if only parameters contain uncertainties (i.e., structured uncertainties). However, these controllers do nothing about unstructured uncertainties which might be the main obstacle of developing high accuracy tracking control of motion systems in some cases and thus exist essential performance degradation, even instability since even a small disturbance may cause the adaptive process instability [14]. As an alternative approach, robust design has also been investigated by some researchers $[15,16]$ to attenuate various uncertainties in physical systems. But parametric uncertainties are not considered 
explicitly in those robust designs, and this will make the robust control too conservative. Sliding mode control in [17] can deal with both parameters uncertainties and unstructured uncertainties. The main drawback is that it utilizes a discontinuous function, that is, sign function, thus causing severe chattering across the sliding surface and even causing instability. Another problem with discontinuous control effort is that no electrical motor can produce discontinuous driving force for compensation in practice.

To handle various uncertainties in one controller, adaptive robust control (ARC) was applied for motion systems driven by linear motors $[5,11]$. Though the ARC controllers proposed in $[5,11]$ can achieve excellent tracking performance in normal working conditions, accurate friction compensation is not considered in these controllers. But for high precision motion performance, the friction problem is one of the significant limitations since friction is a very complicated phenomenon that relies on the physical properties of the contact surfaces such as material property, relative velocity, and lubrication condition in practical applications. A unique tracking error known as quadrant glitch is the result of complex nonlinear friction behavior at motion reversal or nearzero velocity, which will reduce the product quality greatly in manufacture process. In addition, in the low velocity range and during velocity reversal, the nonlinear effects of the friction on the performance of a servo system are known to dominate, compared with those in moderate or steady state velocity range [18]. Failure to compensate nonlinear frictions in applications may lead to large tracking errors, undesired stick-slip motion, and limit cycles when slow velocity is required. Thus the nonlinear frictions encountered in servo systems act often on the main obstacle for high positioning and tracking control performance. From this perspective, when desired trajectory is slow, the performance degradation is inevitable with ARC controllers in $[5,11]$.

In this paper, we will take the friction problem as an important issue which cannot be ignored for high precision motion control, together with other modeling uncertainties. In order to predict and compensate for the underlying friction, many friction models are proposed. Among them, static friction model [19] including stiction, Coulomb friction, viscous friction, and Stribeck effect has been widely accepted. In order to describe the dynamic phenomenon in frictions, many dynamic friction models are proposed. Among these dynamic friction models, in [20], Canudas de Wit et al. proposed the LuGre model, which includes the Stribeck effect, hysteresis, and spring-like characteristics of the stiction, as well as variant break-away force. Owing to its simple structure and capability to capture most of the observed frictional behaviors, LuGre friction model has been widely employed in the literatures $[9,17,21,22]$.

Although LuGre-model-based friction compensation has achieved many successful applications, many researchers still paid lots of attention to static friction model-based control strategies $[10,23,24]$. In fact, there are some practical issues when using LuGre model. Firstly, when the LuGre friction model is used, the state variable representing bristle deformations of the LuGre friction model must be estimated by introducing an estimation process since bristle deformations cannot be measured directly; meanwhile, the uncertainties such as parameter variations and unstructured uncertainties must also be considered to obtain a precise tracking performance. But the nonuniform friction force parameters and normal force parameters cannot be adapted together in one controller [25], and this greatly limits the high precision in servo tracking. Secondly, the LuGre model could become very stiff when the velocity is large, and this may lead to some unavoidable digital implementation problems. For example, it has been reported in [26] that the observer dynamics to recover the unmeasurable internal state of the LuGre model could become unstable at high speed motions, and thus some modifications have to be made [27] which result in a complicated nonlinear controller. Thirdly, a discontinuous function, that is, sign function, was utilized in almost all model based friction compensation techniques $[9,10,17-27]$, certainly also including LuGre-model-based approaches, which may result in high-gain controllers and limit applicability due to the amplification of sensor noise and the occurrence of limit cycles. In addition, as stated above, there is no electrical motor that can produce discontinuous driving force for compensation in practice either. Thus, from practical viewpoints, application engineers and scientists prefer to continuous control efforts to reduce the risk of potential instability.

It is worth to note that experimental measurements have proved that a good static friction model can approximate the real friction force with a degree of $90 \%$ fidelity [28]. It was also shown in [10] that the static friction model and the dynamic friction model predict almost the same friction phenomena induced limit cycles in controlled positioning systems. Hence, static friction model based compensation techniques still have a great significance for practical applications [23].

Based on the limitation analysis of LuGre-model-based friction compensation and meanwhile note the fact that it is more popular when the controller is continuous, simple, less noise-sensitive, and can also achieve high precise performance. With these concerns, a new continuously differentiable friction model proposed in [13] is utilized in this paper to develop a continuous friction compensation law that cancels the majority of friction in the systems in conjunction with adaptive robust control [29] to handle other structured uncertainties and unstructured uncertainties for a motion system directly driven by a dc motor. This new static friction model captures the major effects of friction [13] without involving discontinuous or piecewise continuous functions.

In addition, it is well known that friction models can be applied in either a feedback or a feedforward configuration. A careful design strategy is necessary in a feedback approach to avoid instability. The effectiveness of feedforward depends on the accuracy of the applied friction model. Heuristically, the addition of the model-based desired compensation adaptive robust controller (DCARC) is also developed in this paper following the general procedure outlined in [30] to reduce the overall control effort/chattering and the noise sensitivity problem in application, and thus the tracking accuracy can be further improved. The overall stability is guaranteed by a proper robust feedback control action.

The proposed approaches in this paper present a prescribed output tracking performance in the presence of both 
structured uncertainties and unstructured uncertainties with adequate friction compensation. Moreover, the proposed desired compensation adaptive robust controller has the unique feature that the adaptive model compensation and the regressor depend on the reference output trajectory and online parameter estimates only. Such a structure has several implementation advantages [30]: first, the adaptive model compensation is always bounded when projection type adaption law is used and thus does not affect the closed-loop system stability, as a result, the interaction between the parameter adaptation and the robust control law is reduced, which may facilitate the controller gain tuning process considerably; second, the effect of measurement noise on the adaptive model compensation and on the parameter adaptation law is minimized; consequently, a faster adaptation rate can be chosen in implementation to speed up the transient response and to improve overall tracking performance. To verify the high performance of the proposed controllers, especially in slow tracking conditions, extensive comparative experimental results are obtained for the motion control of a dc motor.

This paper is organized as follows. Section 2 gives the problem formulation and establishes the system dynamic models. Section 3 presents the proposed controller design procedure and its theoretical results. Section 4 carries out the comparative experimental certification. And some conclusions can be found in Section 5 .

\section{Problem Formulation and System Models}

The motor considered here is a current-controlled permanent magnet dc motor with a commercial servo electrical driver directly driving an inertia load [8]. The system is depicted in Figure 1. The goal is to have the inertia load to track any specified smooth motion trajectory as closely as possible.

In general, a drive's dynamics can be divided into inner electrical and outer mechanical subsystems. Due to the physical properties, the outer mechanical subsystem has much slower dynamic response speed in comparison with the inner electrical subsystem. Therefore, the overall performance of the motion control system is determined mostly by the outer mechanical subsystem.

Thus, in the derivation of the model, the current dynamic is neglected in comparison to our interest frequency range due to the much faster electric response. The dynamics of the inertia load can be described by $[8,11]$

$$
\begin{gathered}
m \ddot{y}=K_{i} u-B \dot{y}-F(t, y, \dot{y}), \\
F(t, y, \dot{y}) \triangleq F_{f}(\dot{y})+f(t, y, \dot{y}),
\end{gathered}
$$

where $m$ and $y$ represent the inertial mass of the load and the angular displacement, respectively; $K_{i}$ is the torque constant with respect to the unit of input voltage; $u$ is the control input; $B$ represents the combined coefficient of the modeled damping and viscous friction on the load and the actuator rotor; and $F(t, y, \dot{y})$ represents other disturbances such as static friction effect $F_{f}(\dot{y})$ and uncertain nonlinearities $f(y, \dot{y}, t)$ which cannot be modeled precisely (e.g., external

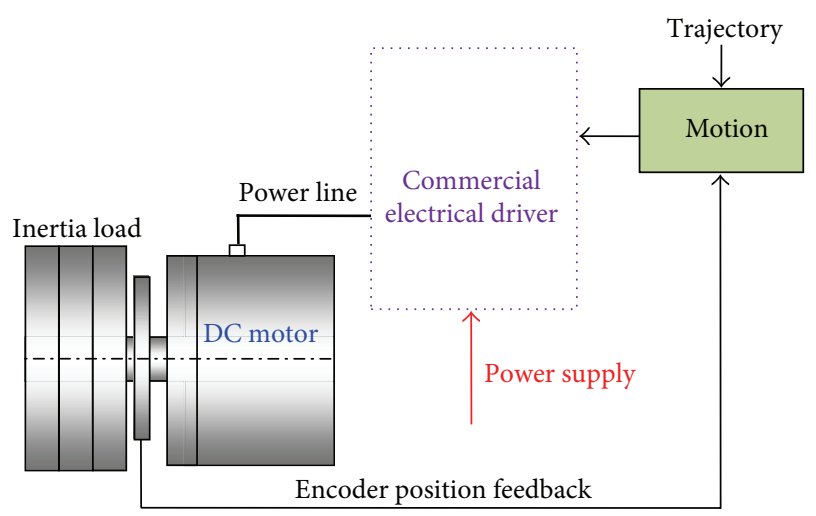

FIGURE 1: The architecture of the positioning motion system driven by DC motor.

cutting forces in machining, unconsidered dynamic friction effects and so on). While there have been many static friction models proposed [19], they are all discontinuous, and thus a continuous static friction model proposed in [13] is employed in this paper to produce continuous compensation effort, which is given by

$$
F_{f}(\dot{y})=l_{1} \tanh \left(s_{1} \omega\right)+l_{2}\left[\tanh \left(s_{2} \omega\right)-\tanh \left(s_{3} \omega\right)\right],
$$

where $\omega$ is the velocity; $l_{1}$ and $l_{2}$ represent different friction levels; and $s_{1}, s_{2}, s_{3}$ denote various shape coefficients to approximate various friction effects.

Remark 1. The friction model in (2) has the following properties [13, 31]: (i) the friction model is continuous differentiable and symmetric about the origin; (ii) the Coulomb friction coefficient is present in the absence of viscous dissipation and is modeled by the term $l_{1} \tanh \left(s_{1} \omega\right)$; (iii) the static coefficient of friction can be approximated by the term $l_{1}+l_{2}$; (iii) the $\operatorname{term} \tanh \left(s_{2} \omega\right)-\tanh \left(s_{3} \omega\right)$ captures the Stribeck effect where the friction coefficient decreases from the static coefficient of friction with increasing slip velocity near the origin.

It is worth to note that the new friction model (2) utilizes the continuous differentiable $\tanh (v)$ function which has the following differential feature with respect to its argument $v$ :

$$
0<\frac{\partial \tanh (v)}{\partial v}<1
$$

The above nice property will be invoked later in our robust controller design to ensure the overall stability of the controlled system.

Based on (1) and (2), we can rewrite the system model (1) in a state-space form as follows:

$$
\begin{gathered}
\dot{x}_{1}=x_{2} \\
\theta_{1} \dot{x}_{2}=u-\theta_{2} S_{f}\left(x_{2}\right)-\theta_{3} x_{2} \\
-\theta_{4}-\theta_{5} P_{f}\left(x_{2}\right)-d(x, t), \\
y=x_{1},
\end{gathered}
$$


where $x=\left[x_{1}, x_{2}\right]^{T}$ represents the state vector of the position and velocity, parameter set $\theta=\left[\theta_{1}, \theta_{2}, \theta_{3}, \theta_{4}, \theta_{5}\right]^{T}$ in which $\theta_{1}=m / K_{i}, \theta_{2}=l_{1} / K_{i}, \theta_{3}=B / K_{i}, \theta_{4}=d_{n} / K_{i}, \theta_{5}=l_{2} / K_{i}$; and $d(x, t)=f(x, t) / K_{i}-d_{n} / K_{i} ; S_{f}\left(x_{2}\right)=\tanh \left(s_{1} x_{2}\right), P_{f}\left(x_{2}\right)=$ $\tanh \left(s_{2} x_{2}\right)-\tanh \left(s_{3} x_{2}\right)$. In (4), a constant $d_{n}$ is employed to denote the nominal value of the lumped disturbance, and thus an adaptive law can be designed for it to learn the slowly changing part of $f(x, t)$ to improve the tracking performance [32].

In general, the system is subjected to structured uncertainties due to large variations in the system parameters, like the variations of $m, K_{i}, l_{1}, l_{2}, B$, and $d_{n}$; that is, parameter set $\theta$ which appears linearly can be unknown due to different system components, working conditions and environments and thus is considered as the structured uncertainty. In addition, $d(x, t)$ is clearly the system unstructured uncertainty.

For most applications, the extents of the structured uncertainty and unstructured uncertainty are known. Thus the following practical assumption is made.

Assumption 2. The extent of the parametric uncertainties and uncertain nonlinearities are known $[8,11]$; that is,

$$
\begin{gathered}
\theta \in \Omega_{\theta} \triangleq\left\{\theta: \theta_{\min } \leq \theta \leq \theta_{\max }\right\}, \\
|d(x, t)| \leq \delta_{d},
\end{gathered}
$$

where $\theta_{\min }=\left[\theta_{1 \min }, \ldots, \theta_{5 \min }\right]^{T}, \theta_{\max }=\left[\theta_{1 \max }, \ldots, \theta_{5 \max }\right]^{T}$, and $\delta_{d}$ are known. In (5), the operation $\leq$ for two vectors is performed in terms of the corresponding elements of the vectors.

Given the desired smooth motion trajectory $y_{d}=x_{1 d}(t)$, the objective is to synthesize a continuous bounded control input $u$ with Assumption 2 such that the output $y=x_{1}$ tracks $x_{1 d}(t)$ as closely as possible in spite of various modeling uncertainties.

\section{Controller Design}

3.1. Discontinuous Projection Mapping and Parameter Adaptation. Let $\widehat{\theta}$ denote the estimate of $\theta$ and $\widetilde{\theta}$ the estimation error (i.e., $\widetilde{\theta}=\widehat{\theta}-\theta$ ). Viewing (5), a discontinuous mapping can be defined as $[11,33]$

$$
\operatorname{Proj}_{\hat{\theta}}\left(\bullet_{i}\right)= \begin{cases}0 & \text { if } \widehat{\theta}_{i}=\theta_{i \max }, \quad \bullet_{i}>0 \\ 0 & \text { if } \hat{\theta}_{i}=\theta_{i \text { min }}, \bullet_{i}<0 \\ \bullet_{i} & \text { otherwise }\end{cases}
$$

where $i=1, \ldots, 5$, by using an adaptation law given by

$$
\dot{\hat{\theta}}=\operatorname{Proj}_{\widehat{\theta}}(\Gamma \tau), \quad \theta_{\min } \leq \widehat{\theta}(0) \leq \theta_{\max },
$$

where $\Gamma>0$ is a diagonal adaptation rate matrix and $\tau$ an adaptation function to be synthesized later. For any adaption function $\tau$, the projection mapping used in (8) guarantees [11, 34]

$$
\begin{aligned}
& \text { (P1) } \hat{\theta} \in \Omega_{\widehat{\theta}} \triangleq\left\{\widehat{\theta}: \theta_{\min } \leq \widehat{\theta} \leq \theta_{\max }\right\}, \\
& \text { (P2) } \widetilde{\theta}^{T}\left(\Gamma^{-1} \operatorname{Proj}_{\widehat{\theta}}(\Gamma \tau)-\tau\right) \leq 0, \quad \forall \tau .
\end{aligned}
$$

3.2. Adaptive Robust Controller (ARC) Design. Define a set of switching functions like quantities as

$$
z_{2}=\dot{z}_{1}+k_{1} z_{1}=x_{2}-x_{2 \mathrm{eq}}, \quad x_{2 \mathrm{eq}} \triangleq \dot{x}_{1 d}-k_{1} z_{1} \text {, }
$$

where $z_{1}=x_{1}-x_{1 d}(t)$ is the output tracking error; $k_{1}$ is a positive feedback gain. Since $G(s)=z_{1}(s) / z_{2}(s)=1 /\left(s+k_{1}\right)$ is a stable transfer function, making $z_{1}$ small or converging to zero is equivalent to making $z_{2}$ small or converging to zero. So the rest of the design is to make $z_{2}$ as small as possible. Differentiating (10) and noting (4), we have

$$
\begin{aligned}
\theta_{1} \dot{z}_{2}= & u-\theta_{1} \dot{x}_{2 \mathrm{eq}}-\theta_{2} S_{f}\left(x_{2}\right)-\theta_{3} x_{2} \\
& -\theta_{4}-\theta_{5} P_{f}\left(x_{2}\right)-d(x, t) .
\end{aligned}
$$

Noting the structure of (11), and based on the adaptive robust design procedure [29], the resulting ARC controller can be given by

$$
\begin{gathered}
u=u_{a}+u_{s}, \\
u_{a}=\widehat{\theta}_{1} \dot{x}_{2 \mathrm{eq}}+\widehat{\theta}_{2} S_{f}\left(x_{2}\right)+\widehat{\theta}_{3} x_{2}+\widehat{\theta}_{4}+\widehat{\theta}_{5} P_{f}\left(x_{2}\right), \\
u_{s}=u_{s 1}+u_{s 2}, \quad u_{s 1}=-k_{2} z_{2},
\end{gathered}
$$

where $k_{2}$ is a positive feedback gain.

In (12), $u_{a}$ functions as adjustable feedforward control law used to achieve an improved model compensation by parameter adaptation (8), and $u_{s}$ functions as a robust control law in which $u_{s 1}$ is a linear robust feedback law to stabilize the nominal model of motion systems and $u_{s 2}$ is nonlinear robust term used to attenuate the effect of model uncertainties as follows. Substituting (12) into (11), we have

$$
\theta_{1} \dot{z}_{2}=-k_{2} z_{2}+u_{s 2}-\widetilde{\theta}^{T} \varphi-d(x, t)
$$

where

$$
\varphi=\left[-\dot{x}_{2 \mathrm{eq}},-S_{f}\left(x_{2}\right),-x_{2},-1,-P_{f}\left(x_{2}\right)\right]^{T}
$$

is the regressor for parameter estimation.

For the robust design, we make the robust term $u_{s 2}$ be any continuous function satisfying the following conditions:

$$
\begin{gathered}
z_{2} u_{s 2} \leq 0 \\
z_{2}\left[u_{s 2}-\widetilde{\theta}^{T} \varphi-d(x, t)\right] \leq \varepsilon_{1},
\end{gathered}
$$

where $\varepsilon_{1}$ is a positive design parameter which can be arbitrarily small. 
Remark 3. Let $h_{1}$ be any smooth function satisfying

$$
h_{1} \geq\left\|\theta_{M}\right\|^{2}\|\varphi\|^{2}+\delta_{d}^{2}
$$

where $\theta_{M}=\theta_{\max }-\theta_{\min }$. Then one smooth example of $u_{s 2}$ satisfying (15) and (16) is given by $[29,34]$

$$
u_{s 2}=-k_{s 1} z_{2} \triangleq-\frac{h_{1}}{2 \varepsilon_{1}} z_{2},
$$

where $k_{s 1}$ is a nonlinear feedback gain.

Other smooth or continuous examples of $u_{s 2}$ can be worked out in the same way as in [29].

Theorem 4. If the adaptation function in (8) is chosen as

$$
\tau=\varphi z_{2}
$$

and choosing feedback gains $k_{1}$ and $k_{2}$ large enough such that the matrix $\Lambda_{1}$ defined below is positive definite (p.d.),

$$
\Lambda_{1}=\left[\begin{array}{cc}
\theta_{1} k_{1} & -\frac{1}{2} \theta_{1} \\
-\frac{1}{2} \theta_{1} & k_{2}
\end{array}\right]
$$

then the proposed ARC control law (12) guarantees the following.

(A) In general, all signals are bounded. Furthermore, the positive definite function $V$ defined by

$$
V=\frac{1}{2} \theta_{1}\left(z_{1}^{2}+z_{2}^{2}\right)
$$

is bounded by

$V(t) \leq \exp \left(-\lambda_{1} t\right) V(0)+\frac{\varepsilon_{1}}{\lambda_{1}}\left[1-\exp \left(-\lambda_{1} t\right)\right]$

where $\lambda_{1}=2 \sigma_{\min }\left(\Lambda_{1}\right) / \theta_{1 \max }$ is the exponentially converging rate, and $\sigma_{\min }(\cdot)$ denotes the minimum eigenvalue of a matrix.

(B) If, after a finite time $t_{0}$, there exist parametric uncertainties only (i.e., $d(x, t)=0$, for any $t \geq t_{0}$ ), in addition to results in (A), asymptotic output tracking is also achieved; that is, $z \rightarrow 0$ as $t \rightarrow \infty$, where $z$ is defined by $z=\left[z_{1}, z_{2}\right]^{T}$.

Proof. See Appendix A.

Remark 5. Results of Theorem 4 indicate that the proposed ARC controller has an exponentially convergence transient performance with the exponentially converging rate $\lambda_{1}$ and the final tracking error (i.e., $V(\infty)$ is bounded by $\left.\varepsilon_{1} / \lambda_{1}\right)$ being able to be adjusted via certain controller parameters freely in a known form. Additionally, condition (20) possesses a great practical advantage that one can use large $k_{1}$ (related to the position measurement) rather than $k_{2}$ (related to the velocity measurement) to retain the overall stability of the controlled system and thus the noise sensitivity can be alleviated.
3.3. Desired Compensation ARC Controller Design. It seems that the control problem with uncertainties has been solved theoretically by ARC controller (12). However, this might have some limitations in practice. Checking the components in the proposed ARC law (12) carefully, it can be seen that the regressor $\varphi$ in the model compensation and the adaptation function $\tau$ (19) depend on state $x$. Such an adaptation structure may have several potential implementation problems $[11,30]$. First, the effect of measurement noise may pollute the regressor $\varphi$, and a slow adaptation rate may have to be used, which in turn reduces the effect of parameter adaptation. Second, the model-based compensation term $u_{a}$ still depends on the actual feedback of the state. Although theoretically the effect of this added implicit feedback loop has been considered in the robust control law design as seen from condition (16), practically, there still exists certain interactions between the model compensation $u_{a}$ and the robust control $u_{s}$. This may complicate the controller gain tuning process in implementation. In [30], a desired compensation adaptive robust control framework is developed by Yao, in which the regressor is calculated by desired trajectory information only. In the following, the desired compensation ARC (DCARC) is applied on our system with the new friction model (2), and some modifications and particular structures of the friction model are utilized to obtain less restrictive conditions on the selection of robust feedback gains to achieve the overall stability.

In order to develop the DCARC law, some modifications of the dynamic of $z_{2}$ in (11) are firstly made as follows:

$$
\begin{aligned}
\theta_{1} \dot{z}_{2}= & u-\theta_{1} \ddot{x}_{1 d}-\theta_{2} S_{f}\left(\dot{x}_{1 d}\right)-\theta_{3} \dot{x}_{1 d}-\theta_{4} \\
& -\theta_{5} P_{f}\left(\dot{x}_{1 d}\right)-d(x, t)-N_{1}-N_{2} \\
& +\left(\theta_{1} k_{1}-\theta_{3}\right) z_{2}-k_{1}\left(\theta_{1} k_{1}-\theta_{3}\right) z_{1},
\end{aligned}
$$

where

$$
\begin{aligned}
& N_{1} \triangleq \theta_{2} S_{f}\left(x_{2}\right)-\theta_{2} S_{f}\left(\dot{x}_{1 d}\right) \\
& N_{2} \triangleq \theta_{5} P_{f}\left(x_{2}\right)-\theta_{5} P_{f}\left(\dot{x}_{1 d}\right)
\end{aligned}
$$

represents the nonlinear approximate errors of the friction.

Noting the property of tanh function (3) and by exploiting the mean value theorem, the following inequalities can be developed for (24):

$$
\begin{gathered}
\left|N_{1}\right| \leq \theta_{2} s_{1}\left|x_{2}-\dot{x}_{1 d}\right| \leq \theta_{2} s_{1}\left|z_{2}\right|+\theta_{2} s_{1} k_{1}\left|z_{1}\right|, \\
\left|N_{2}\right| \leq \theta_{5}\left(s_{2}+s_{3}\right)\left|z_{2}\right|+\theta_{5}\left(s_{2}+s_{3}\right) k_{1}\left|z_{1}\right| .
\end{gathered}
$$

The proposed desired compensation ARC law and the adaptation function have the same form as (12), but with $u_{a}$ determined by the desired trajectory and parameter adaptation only; that is,

$$
\begin{gathered}
u=u_{a}+u_{s}, \\
u_{a}=\widehat{\theta}_{1} \ddot{x}_{1 d}+\widehat{\theta}_{2} s_{f}\left(\dot{x}_{1 d}\right)+\widehat{\theta}_{3} \dot{x}_{1 d}+\widehat{\theta}_{4}+\widehat{\theta}_{5} P_{f}\left(\dot{x}_{1 d}\right), \\
u_{s}=u_{s 1}+u_{s 2}, u_{s 1}=-k_{2} z_{2} .
\end{gathered}
$$


Substituting (26) into (23), one obtains

$$
\begin{aligned}
\theta_{1} \dot{z}_{2}= & -\left(k_{2}-\theta_{1} k_{1}+\theta_{3}\right) z_{2}+u_{s 2}-\widetilde{\theta}^{T} \varphi_{d} \\
& -d(x, t)-N_{1}-N_{2}-k_{1}\left(\theta_{1} k_{1}-\theta_{3}\right) z_{1},
\end{aligned}
$$

where $\varphi_{d}$ is desired regressor given by

$$
\varphi_{d} \triangleq\left[-\ddot{x}_{1 d},-S_{f}\left(\dot{x}_{1 d}\right),-\dot{x}_{1 d},-1,-P_{f}\left(\dot{x}_{1 d}\right)\right]^{T} .
$$

From (27), the robust term $u_{s 2}$ should satisfy the following two conditions:

$$
\begin{gathered}
z_{2} u_{s 2} \leq 0, \\
z_{2}\left[u_{s 2}-\widetilde{\theta}^{T} \varphi_{d}-d(x, t)\right] \leq \varepsilon_{2},
\end{gathered}
$$

where $\varepsilon_{2}$ is a positive design parameter which can be arbitrarily small.

An example of $u_{s 2}$ satisfying (29) and (30) is given as follows:

$$
u_{s 2}=-k_{s 2} z_{2} \triangleq-\frac{h_{2}}{2 \varepsilon_{2}} z_{2},
$$

where $k_{s 2}$ is a nonlinear feedback gain and $h_{2}$ satisfies

$$
h_{2} \geq\left\|\theta_{M}\right\|^{2}\left\|\varphi_{d}\right\|^{2}+\delta_{d}^{2}
$$

Theorem 6. With the projection type adaptation law (8) in which adaptation function is given as $\tau=\varphi_{d} z_{2}$ and choosing suitable feedback gains $k_{1}$ and $k_{2}$ such that the matrix $\Lambda_{2}$ defined below is positive definite,

$$
\Lambda_{2}=\left[\begin{array}{cc}
\theta_{1} k_{1} & -\frac{1}{2} \eta \\
-\frac{1}{2} \eta & k_{2}-\theta_{1} k_{1}+\theta_{3}-\theta_{2} s_{1}-\theta_{5}\left(s_{2}+s_{3}\right)
\end{array}\right],
$$

where $\eta=\left|\theta_{1}-k_{1}\left(\theta_{1} k_{1}-\theta_{3}\right)\right|+\theta_{2} k_{1} s_{1}+\theta_{5} k_{1}\left(s_{2}+s_{3}\right)$, then the proposed desired compensation ARC law (26) guarantees the following.

(A) In general, all signals are bounded. Furthermore, the positive definite $V$ is bounded by

$$
V(t) \leq \exp \left(-\lambda_{2} t\right) V(0)+\frac{\varepsilon_{2}}{\lambda_{2}}\left[1-\exp \left(-\lambda_{2} t\right)\right],
$$

where $\lambda_{2}=2 \sigma_{\min }\left(\Lambda_{2}\right) / \theta_{1 \max }$ is the exponentially converging rate.

(B) If, after a finite time $t_{0}, d(x, t)=0$, in addition to results in (A), asymptotic output tracking is also achieved; that is, $z \rightarrow 0$ as $t \rightarrow \infty$.

Proof. See Appendix B.

Remark 7. Results of Theorem 6 indicate that the proposed desired compensation ARC controller (26) has the same performance properties as the previous ARC controller (12) stated in Remark 5. Furthermore, the DCARC law (26) also

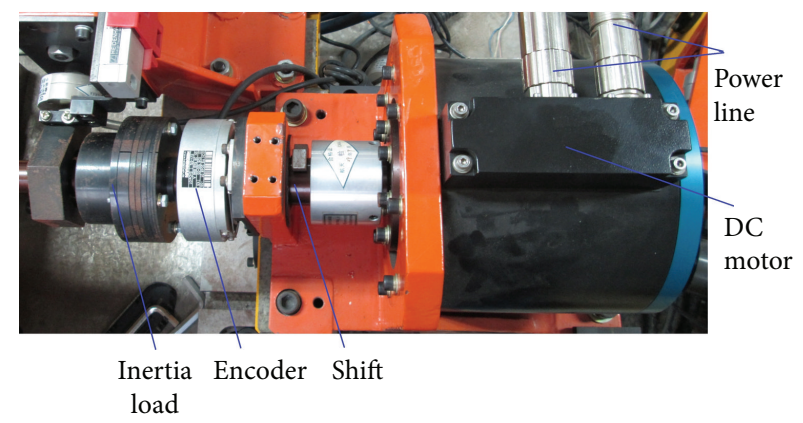

FIGURE 2: Experimental platform of DC motor driven system.

has the following advantages [30]. (i) Since the regressor $\varphi_{d}$ depends on the reference trajectory only, it is bounded and can be calculated offline to save online computation time if needed. (ii) Due to the use of projection mapping in (8), the parameter estimation is bounded as shown by P1 of (9). Thus the model compensation $u_{a}$ in (26) is bounded no matter what type of adaptation law is going to be used. This implies that $u_{a}$ does not affect the closed-loop system stability at all and the robust control function $u_{s}$ can be synthesized totally independent of the design of parameter adaptation law for robust stability. (iii) Gain tuning process becomes simpler since some of the bounds like the bound of the first term inside the bracket of the left hand side of (30) can be estimated offline. (iv) The effect of measurement noise is reduced.

Remark 8. It is worth to note that the condition on the selection of robust feedback gain $k_{2}$ is much less restrictive than those in [30], which can further alleviate the effect of noisy velocity feedback. This is achieved by judiciously selecting a p.d. function given by (21) instead of the general formulations in $[30]$.

\section{Comparative Experimental Results}

4.1. Experiment Setup. To illustrate the above designs and study fundamental problems associated with the high accuracy control of dc motor motion systems, a verification platform has been set up shown in Figure 2. The detail description of the hardware components used in the platform can be found in [8] and the measurement and control system consists of monitoring software and real time control software (more details can be found in [35]). The sampling time is $0.5 \mathrm{~ms}$.

4.2. System Identification. Due to shape coefficients $s_{1}, s_{2}$, and $s_{3}$ existing in our friction models and our proposed controllers, the static friction effects have to be identified. The following experimental friction model identification procedure is taken: first, a series of control constant velocity trajectory is applied to the servo system. Steady signals are finally recorded to obtain the static mapping relationship between the control input $u$ and the output velocity signal. From (4), when the steady output velocity is constant (i.e., the inertial force is zero), the control input $u$ is equal to the normalized friction 


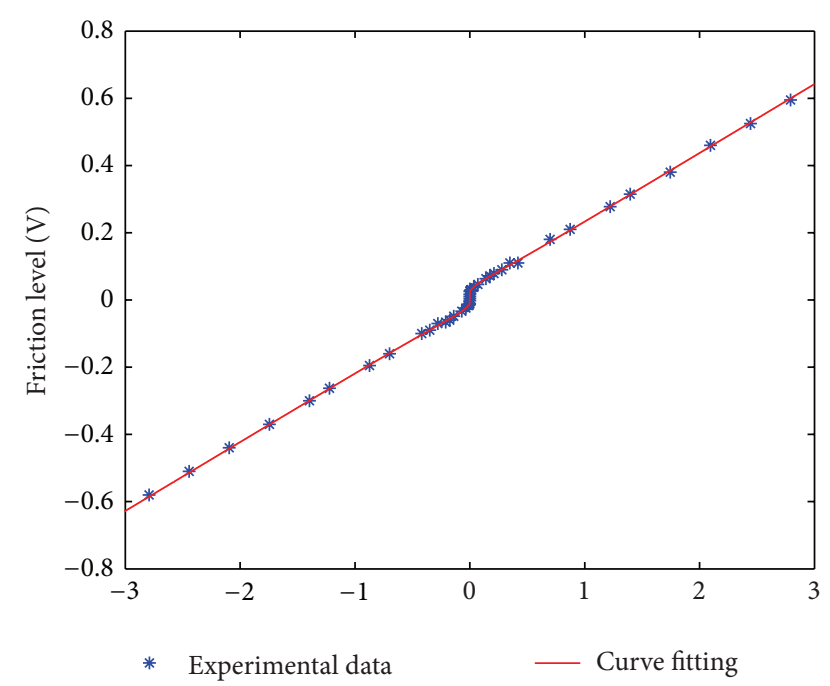

(a) The overall friction identification data

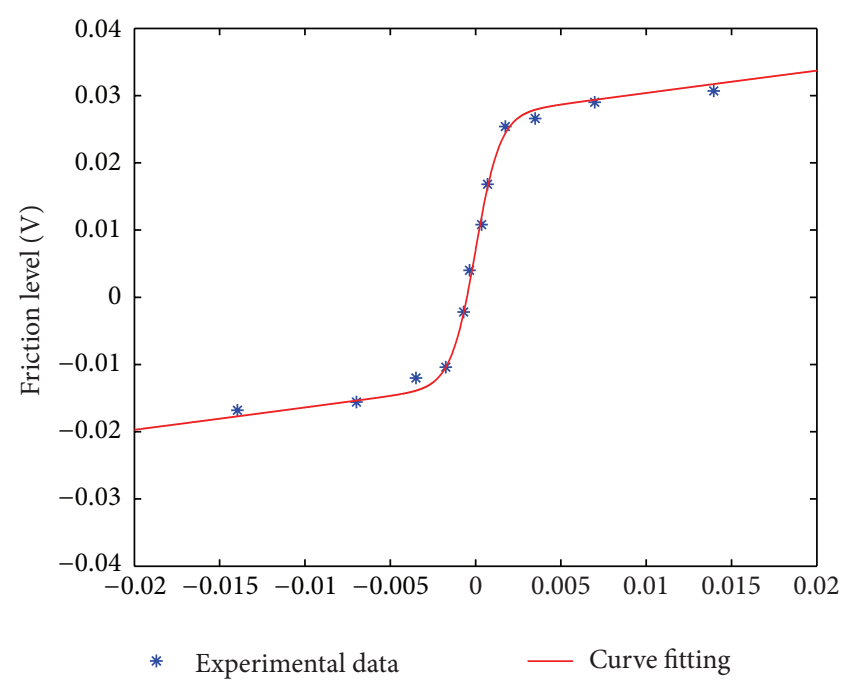

(b) The zoom figure at zero velocity region in (a)

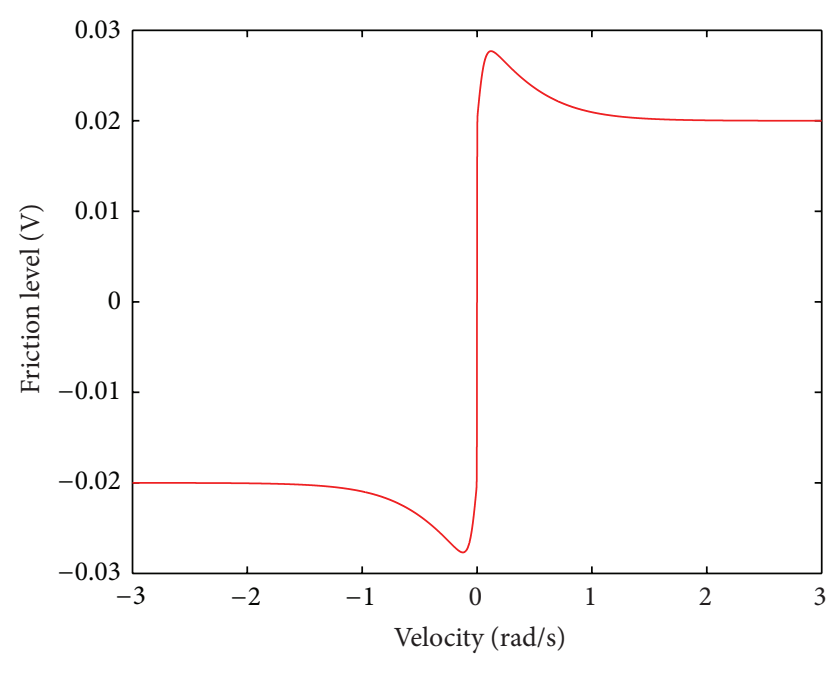

- Identified Stribeck effect

(c) The obtained static friction described in (2) with $s_{1}=700, s_{2}=15$, and $s_{3}=1.5$; the normalized friction level $l_{1} / K_{i}=0.02, l_{2} / K_{i}=0.01$

FIgURE 3: Experimental results and curve fitting of nonlinear friction. level. The result of static mapping relationship identified by experiments is shown in Figure 3, and the structure model of the friction is obtained by polynomial curve-fitting method. The corresponding coefficients $s_{1}=700, s_{2}=15$, and $s_{3}=$ 1.5 are used to fit the experimental curve in Figure 3, and these coefficients will be utilized in our proposed controllers. Though the normalized friction level parameters are also obtained, they are not used since they may vary in practice, and will be adapted by the designed adaptive law.

4.3. Comparative Experimental Results. The following five controllers are compared to verify the effectiveness of the proposed control schemes in the next experiments.

(1) ARCF: this is the adaptive robust controller (12) with static friction model (2) proposed in this paper and described in previous sections. As in [11], for simplicity, the control function $u_{s}=u_{s 1}+u_{s 2}$ is implemented as $u_{s}=-k^{\prime} z_{2}$ by choosing a sufficiently large nonlinear feedback gain $k^{\prime}$ to satisfy (16). The control gains are chosen as $k_{1}=240, k^{\prime}=5$, which are tuned via online try-and-error method, and some statements about gain choosing can be found in [11, 36]. The bounds of parametric ranges are given by $\theta_{\min }=$ $[0,0,0,-0.01,0]^{T}, \theta_{\max }=\left[5 \times 10^{-3}, 0.1,0.4,0.01\right.$, $0.5]^{T}$. The initial estimate of $\theta$ is chosen as $[1.9 \times$ $\left.10^{-3}, 1 \times 10^{-3}, 0.0353,0,0.02\right]^{T}$. Adaptation rates are set at $\Gamma=\operatorname{diag}\{0.1,0.5,2,0.3,0.5\}$.

(2) DCARCF: this is the desired compensation adaptive robust controller (26) with static friction model (2), proposed in this paper and described in previous sections. For fair comparison, all gains used are chosen same as corresponding gains of the ARCF controller but with large adaptation rates $\Gamma=\operatorname{diag}\{0.3$, $1,3,0.5,1\}$ according to Remark 7 .

(3) ARC: this is the adaptive robust controller only with approximate continuous Coulomb friction model. The controller can be outlined from [11, 29]. In our experiments, the ARC controller is implemented same as ARCF but without $\theta_{5}$ adaptation (i.e., no Stribeck effect), and thus, the gains are chosen same as corresponding gains of the ARCF controller but with $\Gamma=\operatorname{diag}\{0.1,0.5,2,0.3,0\}$.

(4) DCARC: this is the desired compensation adaptive robust controller only with approximate continuous Coulomb friction model. The controller can be outlined from [11, 30]. In our experiments, the DCARC controller is implemented same as DCARCF but without $\theta_{5}$ adaptation, and thus, the gains are chosen same as corresponding gains of the DCARCF controller but with $\Gamma=\operatorname{diag}\{0.3,1,3,0.5,0\}$.

(5) PID: the proportional-integral-derivative controller, which is commonly used in industrials which can be treated as a reference controller for comparison. The controller parameters are $k_{P}=120, k_{I}=60$, and $k_{D}=$ 0 , which represent the $P$-gain, $I$-gain and $D$-gain, 
respectively. These controller gains are tuned carefully via error-and-try method. One may argue that the larger parameters can make better tracking performance. But these parameters are achieved ultimately and larger parameters will lead the system to unstable due to measurement noise and/or unmodeled dynamics. Thus using the PID controller with these parameters to compare with the proposed controllers is fair.

The following five performance indexes will be used to measure the quality of each control algorithm, that is, the maximum, average, and standard deviation of the tracking error, average control input, and normalized control variations. Their definitions are made as follows.

(1) Maximal absolute value of the tracking errors is defined as

$$
M_{e}=\max _{i=1, \ldots, N}\left\{\left|z_{1}(i)\right|\right\},
$$

where $N$ is the number of the recorded digital signals and is used as an index of measure of tracking accuracy.

(2) Average tracking error is defined as

$$
\mu=\frac{1}{N} \sum_{i=1}^{N}\left|z_{1}(i)\right|
$$

and is used as an objective numerical measure of average tracking performance.

(3) Standard deviation performance index is defined as

$$
\sigma=\sqrt{\frac{1}{N} \sum_{i=1}^{N}\left[\left|z_{1}(i)\right|-\mu\right]^{2}}
$$

to measure the deviation level of tracking errors.

(4) Average control input index is defined as

$$
L_{u}=\sqrt{\frac{1}{N} \sum_{i=1}^{N}|u(i)|^{2}}
$$

and is used to evaluate the amount of control effort.

(5) Normalized control variations is defined as

$$
L_{c}=\frac{L_{\Delta u}}{L_{u}}
$$

and is used to measure the degree of control chattering, where

$$
L_{\Delta u}=\sqrt{\frac{1}{N} \sum_{i=1}^{N}|u(i \Delta T)-u((i-1) \Delta T)|^{2}},
$$

where $\Delta T$ is the time interval.

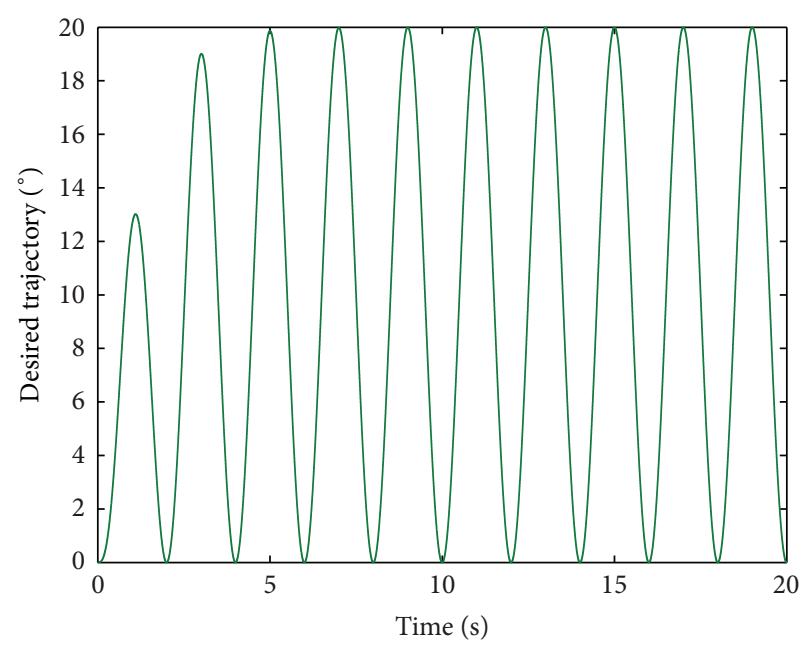

FIgURE 4: The desired trajectory (normal case).
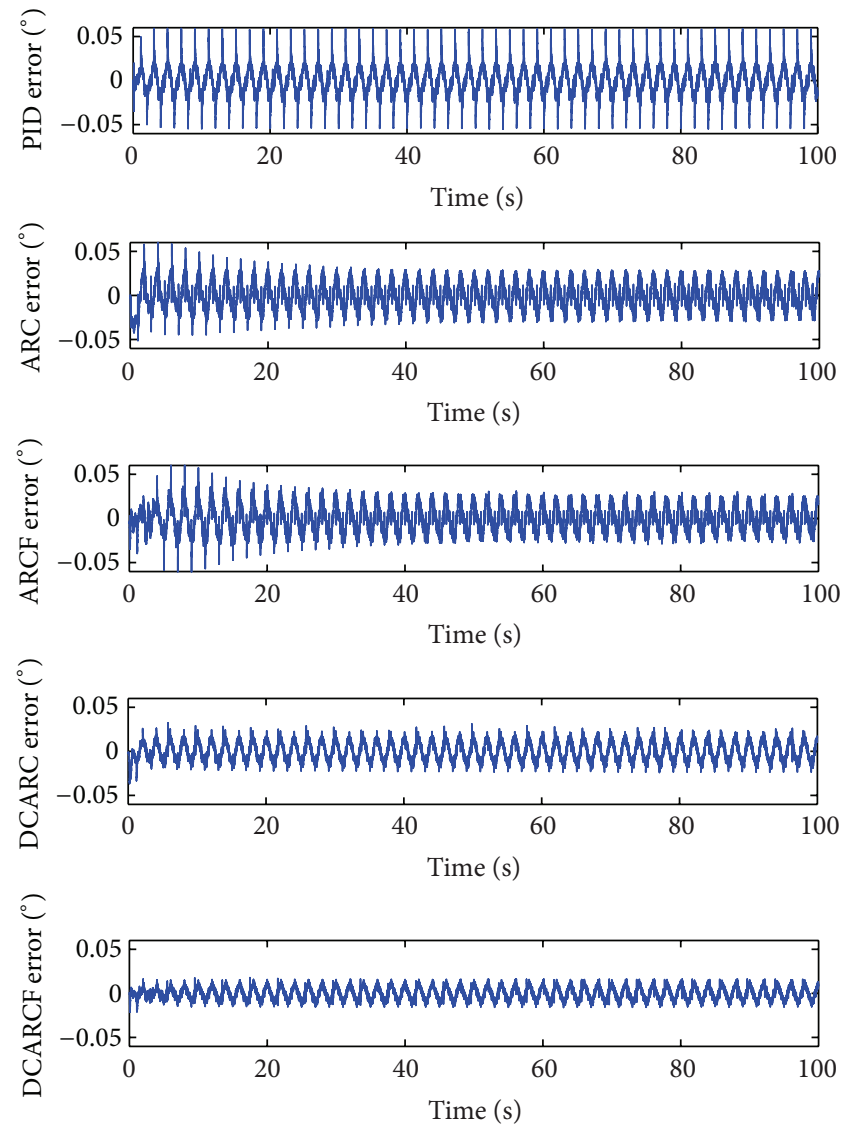

FIGURE 5: Tracking errors of five controllers in normal case, PID, ARC, ARCF, DCARC, and DCARCF, from top to bottom, respectively.

To verify the performance of the proposed controllers, the five controllers are first tested for a sinusoidal-like motion trajectory $x_{1 d}=10[1-\cos (3.14 t)][1-\exp (-0.5 t)]^{\circ}$ which ensures the desired trajectory smooth enough. The desired motion trajectory is shown in Figure 4. The corresponding tracking errors under five controllers are shown in Figure 5, 

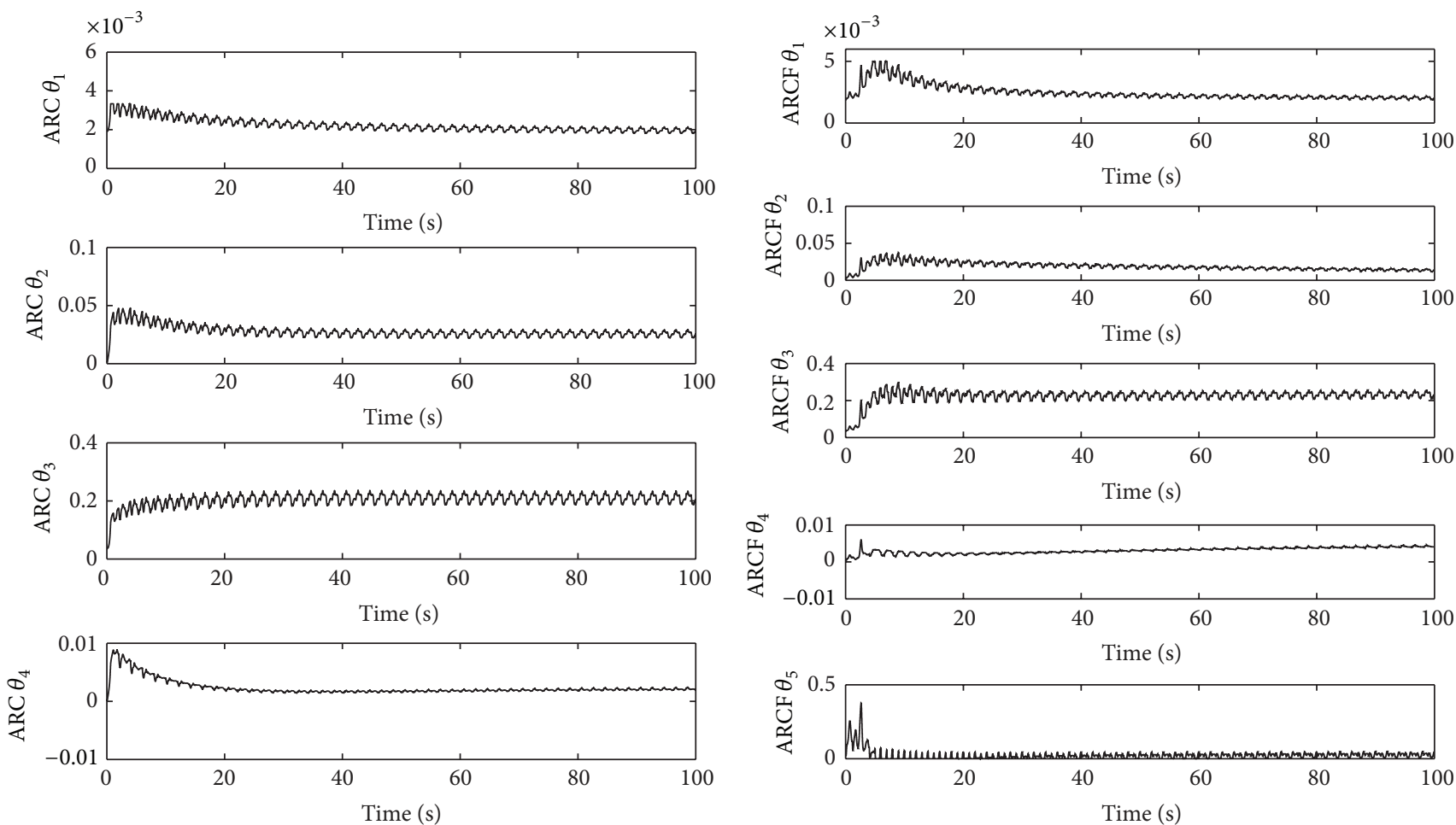

(a) The parameter estimation of ARC controller

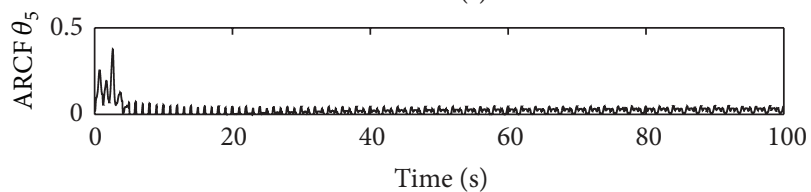

(b) The parameter estimation of ARCF controller
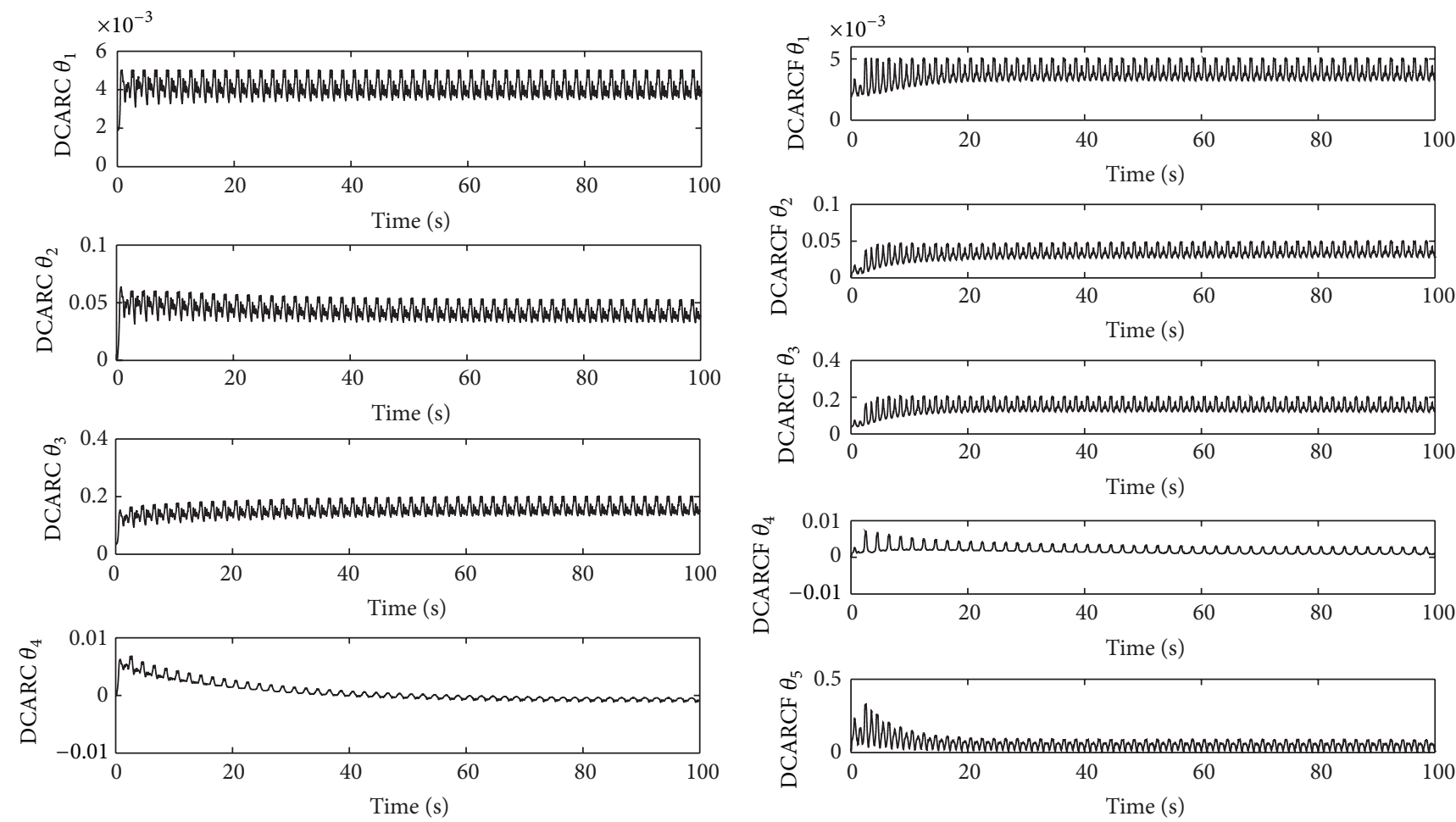

(c) The parameter estimation of DCARC controller
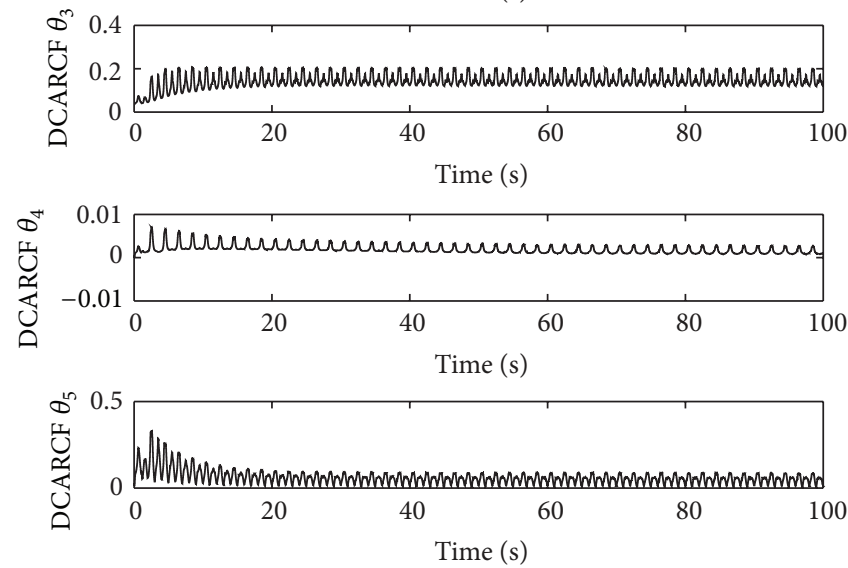

(d) The parameter estimation of DCARCF controller

Figure 6: Parameter estimations of five controllers in normal case. 
TABLE 1: Performance indexes during the last two seconds.

\begin{tabular}{lccccc}
\hline Indexes & $M_{e}$ & $\mu$ & $\sigma$ & $L_{u}$ & $L_{c}$ \\
\hline PID & 0.0581 & 0.0132 & 0.0106 & 0.1101 & 0.0154 \\
ARC & 0.0283 & 0.0125 & 0.0076 & 0.1061 & 0.0138 \\
ARCF & 0.0260 & 0.0122 & 0.0073 & 0.1060 & 0.0141 \\
DCARC & 0.0234 & 0.0091 & 0.0046 & 0.1064 & 0.0117 \\
DCARCF & 0.0152 & 0.0059 & 0.0034 & 0.1059 & 0.0117 \\
\hline
\end{tabular}
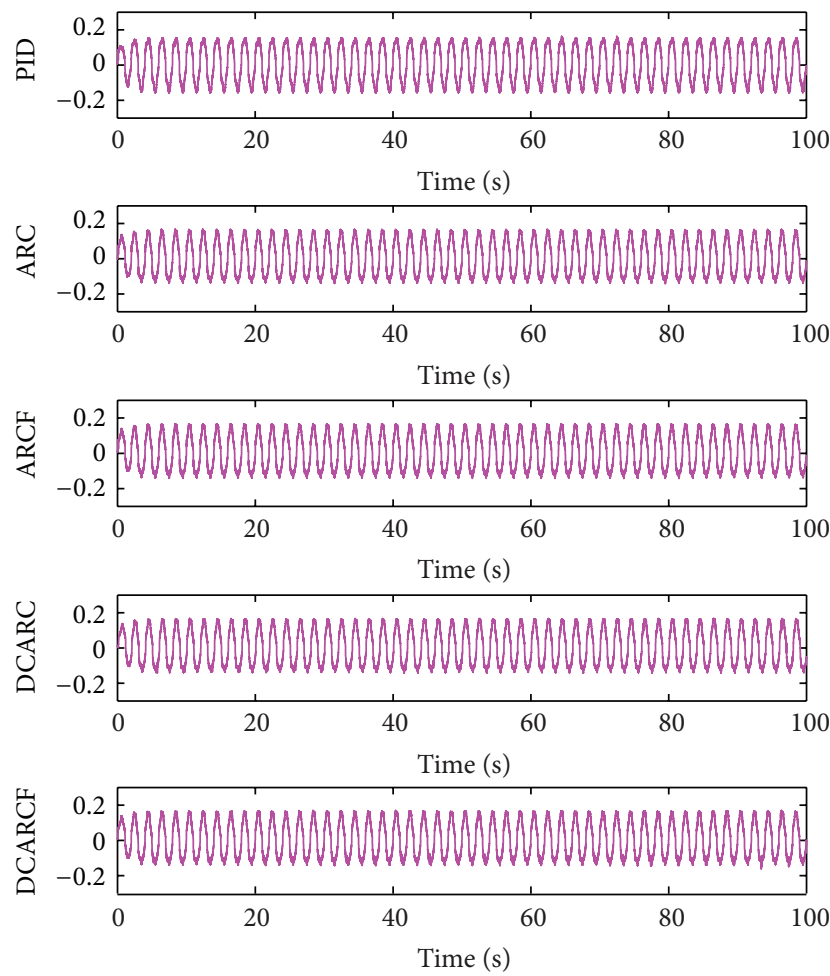

FIGURE 7: Control inputs of five controllers in normal case (the ordinate unit is $\mathrm{V}$ ).

respectively. As seen, the proposed DCARCF controller has a better performance than those of the other four controllers in terms of both transient and final tracking errors since the DCARCF controller both employed the new continuous friction model to achieve accurate friction compensation and large adaptation rate, and then the resulting learning capability is enhanced; while ARCF also employed the explicit friction model and thus obtained better performance than ARC and PID, the tracking process is chattering since its regressor depends on the noisy actual states, and that is the reason it is worse than the DCARC controller. The PID controller just has some robustness with respect to uncertainties and their tracking errors are relatively large. Moreover, by using the parameter adaptation as shown in Figure 6, the final tracking error of DCARCF is reduced almost down to $0.015^{\circ}$ while PID has large quadrant glitch (about $0.06^{\circ}$ ) due to the large friction disturbance during velocity reversal. This illustrates the effectiveness of using the adjustable desired compensation technique and the new friction model which

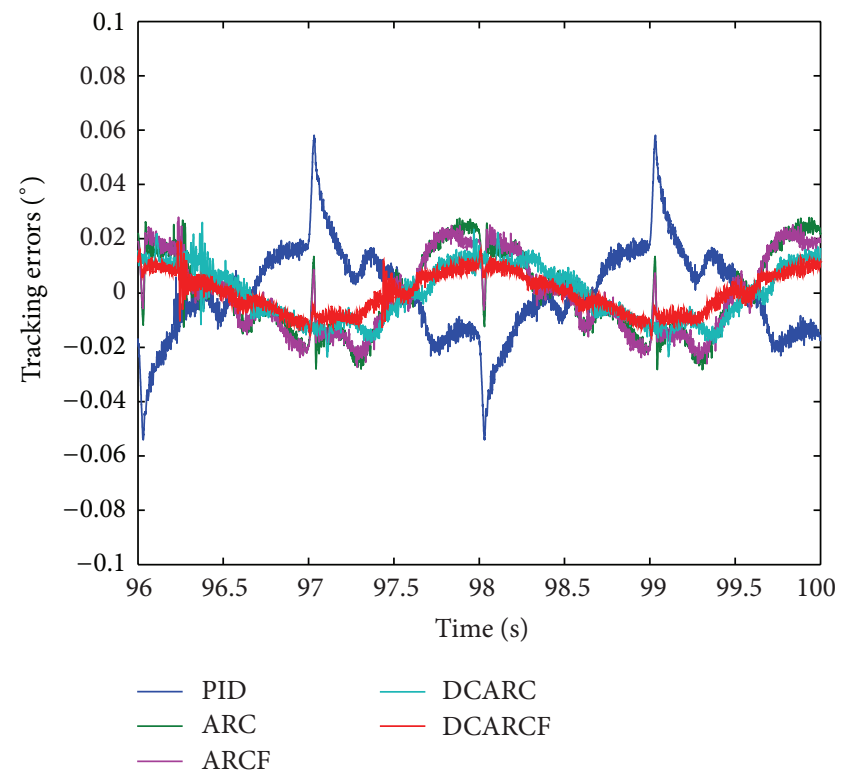

FIGURE 8: Tracking errors of five controllers during the last two cycles.

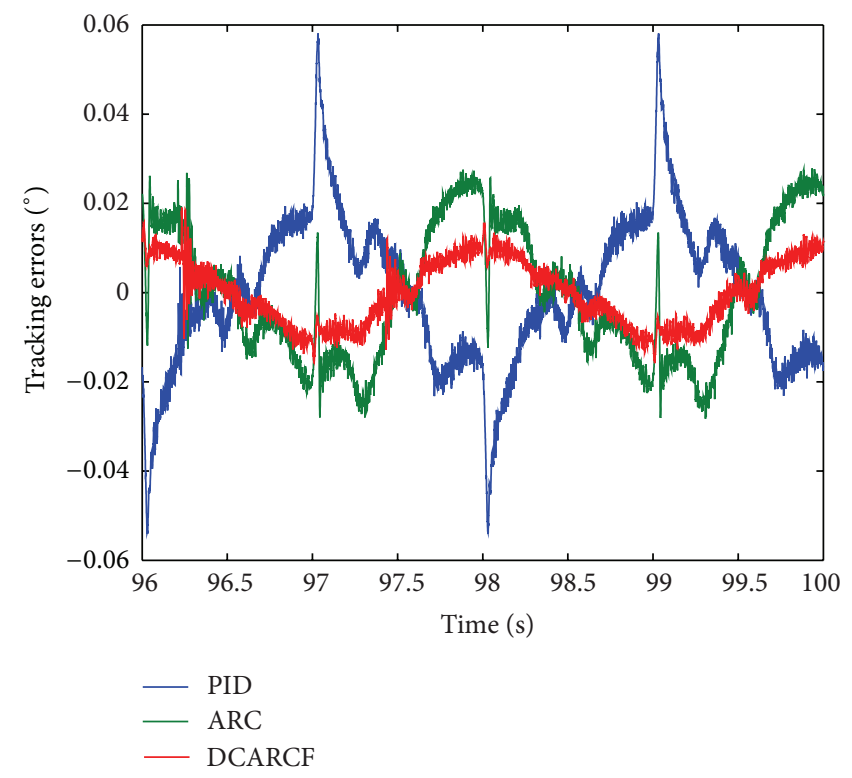

FIGURE 9: Tracking errors of three controllers during the last two cycles.

can effectively overcome the nonlinear friction disturbance in practice. The control inputs of five controllers are shown in Figure 7, which is continuous, regular, and bounded. To investigate the final tracking accuracy, the last two cycles' tracking errors are present in Figure 8, and a more clear comparison is given in Figure 9, in which only PID, ARC, and DCARCF are compared. The experimental results in terms of performance indexes are given in Table 1 during the last two seconds. As seen from the table, DCARCF performs best performance in all indexes, compared with the other 
TABle 2: Performance indexes during the last two seconds in slow tracking case.

\begin{tabular}{lccccc}
\hline Indexes & $M_{e}$ & $\mu$ & $\sigma$ & $L_{u}$ & $L_{c}$ \\
\hline PID & 0.0247 & 0.0032 & 0.0050 & 0.0392 & 0.0196 \\
ARC & 0.0111 & 0.0032 & 0.0022 & 0.0389 & 0.0170 \\
ARCF & 0.0099 & 0.0024 & 0.0021 & 0.0389 & 0.0188 \\
DCARC & 0.0101 & 0.0016 & 0.0023 & 0.0382 & 0.0172 \\
DCARCF & 0.0077 & 0.0012 & 0.0014 & 0.0371 & 0.0171 \\
\hline
\end{tabular}

four controllers. It is worth to note that the PID controller exhibits worst tracking performance but consumes maximal control efforts and chattering, which can indicate that PID has reached its limitations in this tracking task.

To further test the performance of the proposed algorithms, a slow motion trajectory $x_{1 d}=[1-$ $\cos (3.14 t)][1-\exp (-0.5 t)]^{\circ}$ shown in Figure 10 is used. In this test stage, the desired velocity is reduced to only a tenth of the previous one, and thus the effects of the nonlinear friction are exaggerated and may be the dominate factor effecting the tracking performance. The tracking errors of the five controllers are shown in Figure 11, respectively. As seen, even for such a slow tracking experiment under strong nonlinear friction, the proposed DCARCF controller is able to compensate the modeled nonlinear friction and attenuate unmodeled effects and an improved performance is achieved in comparison to the other four controllers. The parameter estimation and control inputs are omitted with space limitations since they are regular and bounded. More clear comparison is presented in Figures 12 and 13. The performance indexes with this case are collected in Table 2. From these results, again, it can be seen that the proposed DCARCF controller has the best tracking performance.

\section{Conclusion}

In this paper, with a new static friction model capable of capturing major nonlinear friction effects, a continuous ARC controller and a continuous desired compensation ARC controller have been developed for high-performance robust motion systems directly driven by dc motors. The proposed controllers take into account the effect of model uncertainties coming from the inertia load, modeled friction force, and unmodeled disturbances. In addition, by using the nice differential property of the new friction model and integrating the mean value theorem, a proper robust feedback term is synthesized in our proposed desired compensation ARC controller, which ensures the overall stability while reduces restrictive conditions. Theoretically, the resulting controllers guarantee a prescribed transient performance and final tracking accuracy in general while achieving asymptotic tracking in the presence of parametric uncertainties. Furthermore, the desired compensation ARC scheme offers several implementation advantages such as reduced effect of measurement noise and a faster adaptation rate in implementation. Comparative experiments are carried out for the motion control of a dc motor to illustrate the high performance of

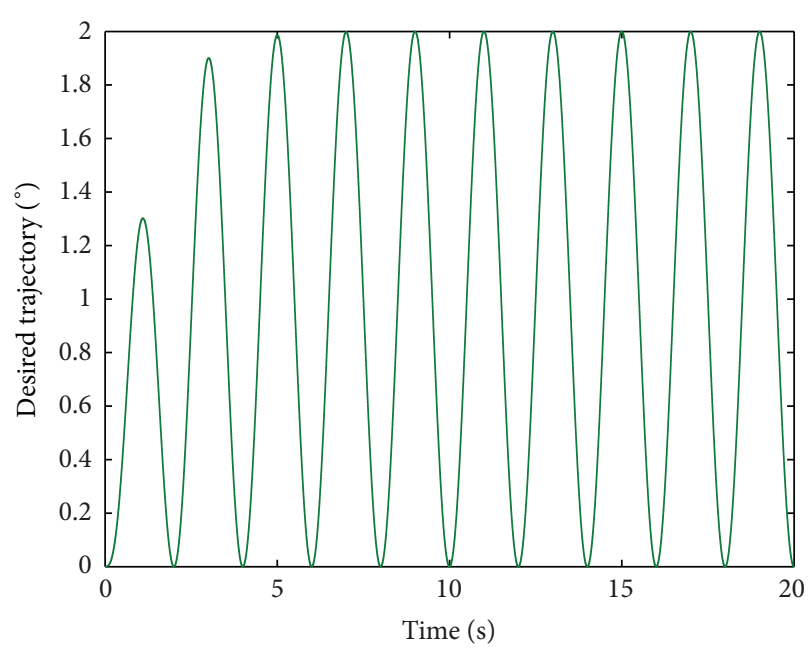

FIgURE 10: The desired trajectory (slow case).
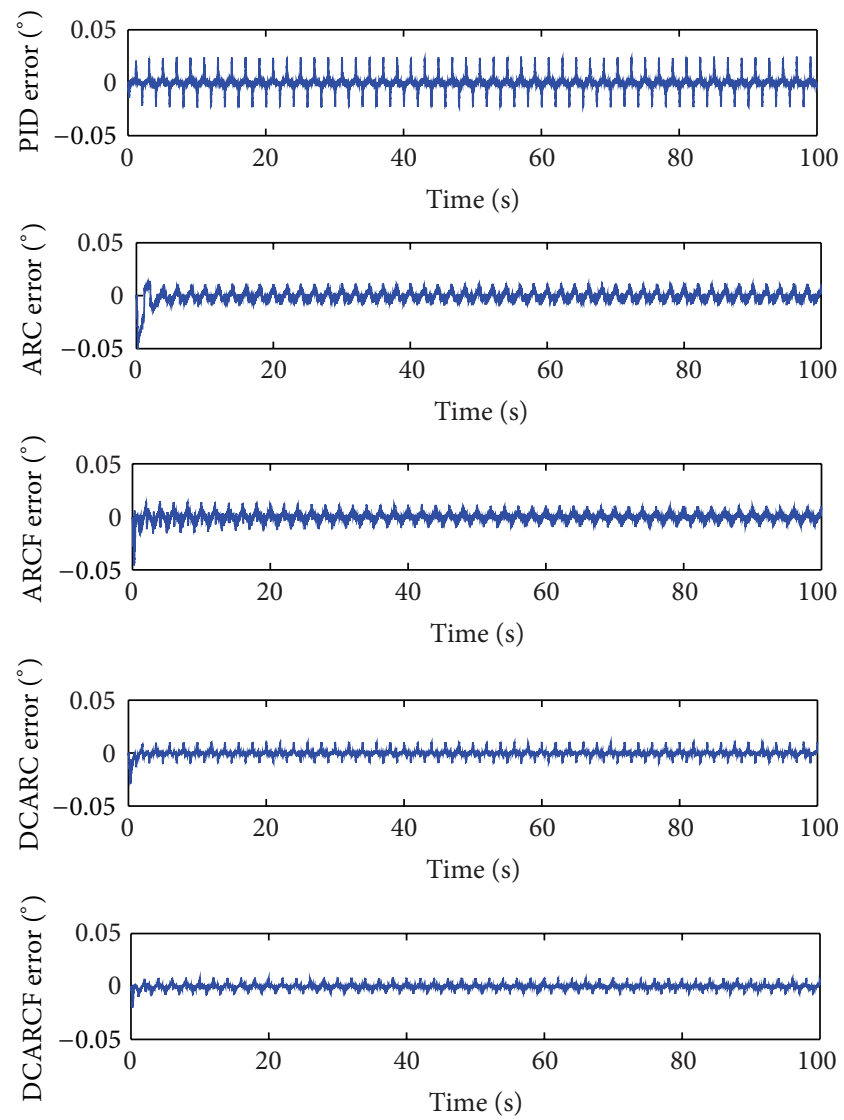

FIGURE 11: Tracking errors of five controllers in slow tracking case.

the proposed ARC strategies. Comparative experimental results show that smaller tracking error and smoother control effort are obtained using the ARC algorithms with the continuous friction model than with approximate Coulomb friction only, demonstrating the effectiveness of the proposed algorithms in practical applications. As future works, it is interesting to consider other applications of the proposed scheme, such as the control of transport problem [37]. 


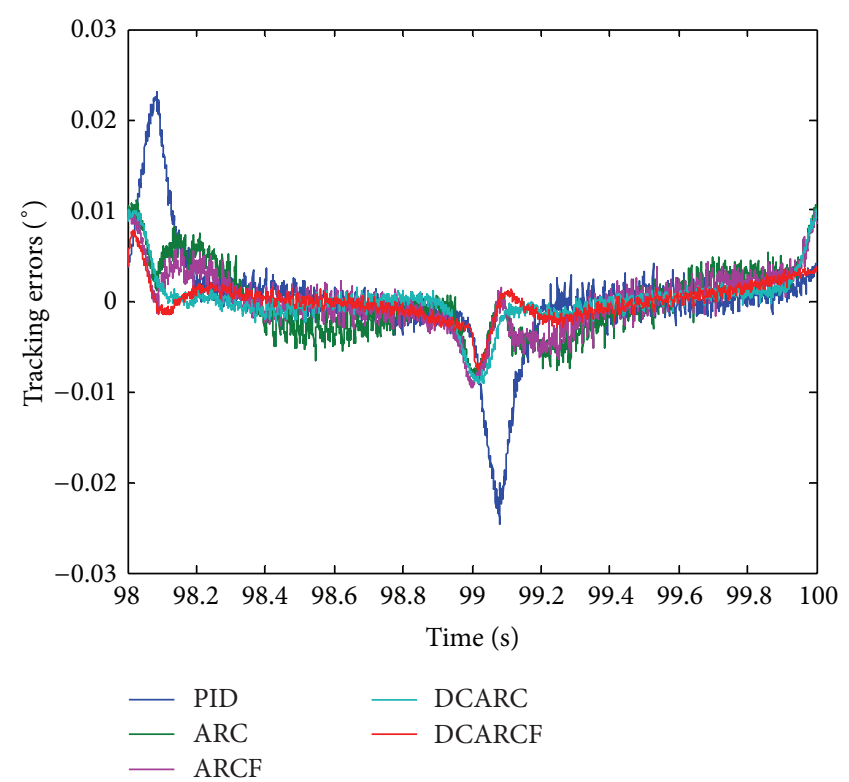

FIGURE 12: Tracking errors of five controllers during the last cycle in slow tracking case.

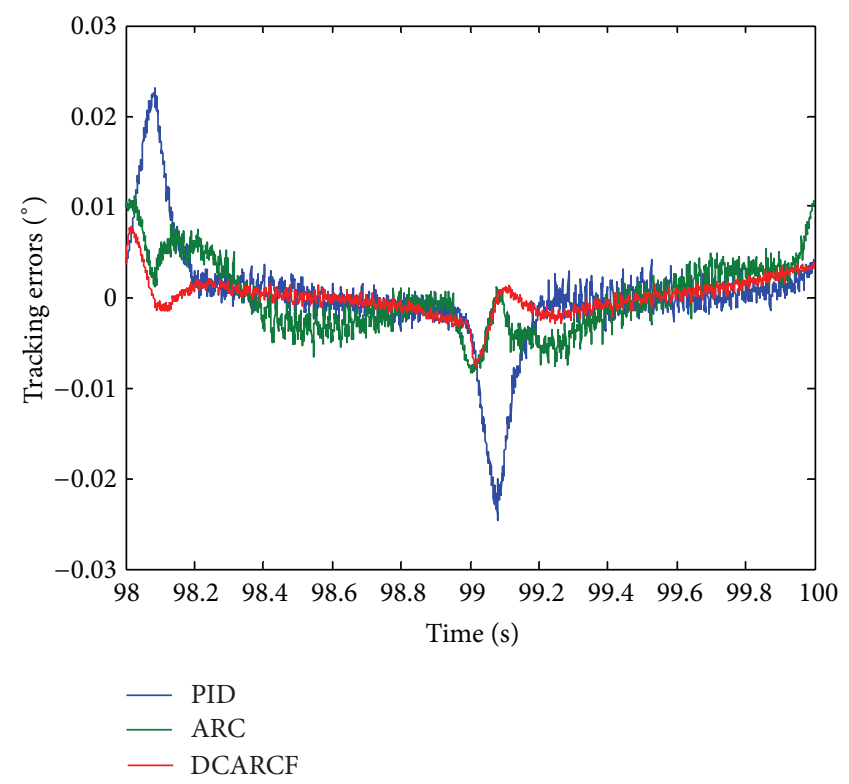

FIGURE 13: Tracking errors of three controllers during the last cycle in slow tracking case.

\section{Appendices}

\section{A. Proof of Theorem 4}

From (13) and noting (10), the time derivative of $V$ can be given by

$$
\begin{aligned}
\dot{V} & =\theta_{1} z_{1} \dot{z}_{1}+\theta_{1} z_{2} \dot{z}_{2} \\
& =\theta_{1} z_{1}\left(z_{2}-k_{1} z_{1}\right)-k_{2} z_{2}^{2}+z_{2}\left[u_{s 2}-\widetilde{\theta}^{T} \varphi-d(x, t)\right] .
\end{aligned}
$$

Using the condition in (16), we have

$$
\dot{V} \leq-z^{T} \Lambda_{1} z+\varepsilon_{1} .
$$

Noting that the matrix $\Lambda_{1}$ defined in (20) is positive definite and based on the definition of $\lambda_{1}$, we can infer

$$
\dot{V} \leq-\lambda_{1} V+\varepsilon_{1}
$$

which leads to (22) by using comparison lemma [38]. Thus, $z_{1}$ and $z_{2}$ are bounded which means the state $x$ is bounded. $S_{f}\left(x_{2}\right)$ and $P_{f}\left(x_{2}\right)$ are bounded from their definition. From property P1 in (9), all estimated parameters are bounded, and thus the control input $u$ is bounded. This proves that all signals in the closed loop systems are bounded and the results in (A) of Theorem 4 are proved.

Now for part (B), when $d(x, t)=0$, choose a p.d. function $V_{s}$ as

$$
V_{s}=V+\frac{1}{2} \widetilde{\theta}^{T} \Gamma^{-1} \widetilde{\theta}
$$

From (13) with $d(x, t)=0$, the condition in (15), and the definition of $\tau$ in (19), the derivative of $V_{s}$ satisfies

$$
\begin{aligned}
\dot{V}_{s} & =\theta_{1} z_{1}\left(z_{2}-k_{1} z_{1}\right)-k_{2} z_{2}^{2}+z_{2}\left[u_{s 2}-\widetilde{\theta}^{T} \varphi\right]+\widetilde{\theta}^{T} \Gamma^{-1} \dot{\hat{\theta}} \\
& \leq-z^{T} \Lambda_{1} z+\widetilde{\theta}^{T}\left(\Gamma^{-1} \dot{\hat{\theta}}-\tau\right) .
\end{aligned}
$$

Noting the property P2 of (9), we have [36]

$$
\dot{V}_{s} \leq-\sigma_{\min }\left(\Lambda_{1}\right)\left(z_{1}^{2}+z_{2}^{2}\right) \triangleq W .
$$

Therefore, $W \in L_{2}$ and $V_{s} \in L_{\infty}$. Since all signals are bounded, from (10) and (13), it is easy to check that $\dot{W}$ is bounded and thus uniformly continuous. By Barbalat's lemma, $W \rightarrow 0$ as $t \rightarrow \infty$ [36], which leads to (B) of Theorem 4 .

\section{B. Proof of Theorem 6}

The proof is similar to that of Theorem 4. From (27), the time derivative of $V$ can be given by

$$
\begin{aligned}
\dot{V}= & \theta_{1} z_{1} \dot{z}_{1}+\theta_{1} z_{2} \dot{z}_{2} \\
= & \theta_{1} z_{1}\left(z_{2}-k_{1} z_{1}\right)-k_{1}\left(\theta_{1} k_{1}-\theta_{3}\right) z_{1} z_{2} \\
& -\left(k_{2}-\theta_{1} k_{1}+\theta_{3}\right) z_{2}^{2}+z_{2}\left[u_{s 2}-\widetilde{\theta}^{T} \varphi_{d}-d(x, t)\right] \\
& -N_{1} z_{2}-N_{2} z_{2} .
\end{aligned}
$$

Using the condition in (30) and noting (25), we have

$$
\begin{aligned}
\dot{V} \leq & -k_{1} \theta_{1} z_{1}^{2}+\left[\theta_{1}-k_{1}\left(\theta_{1} k_{1}-\theta_{3}\right)\right] z_{1} z_{2} \\
& -\left(k_{2}-\theta_{1} k_{1}+\theta_{3}\right) z_{2}^{2}+\varepsilon_{2}+\theta_{2} s_{1} z_{2}^{2}+\theta_{2} s_{1} k_{1}\left|z_{1} z_{2}\right| \\
& +\theta_{5}\left(s_{2}+s_{3}\right) z_{2}^{2}+\theta_{5}\left(s_{2}+s_{3}\right) k_{1}\left|z_{1} z_{2}\right| .
\end{aligned}
$$


Noting (33), since $\Lambda_{2}$ is positive-definite, thus the following inequation is true:

$$
\begin{aligned}
\dot{V} & \leq-\sigma_{\min }\left(\Lambda_{2}\right)\left(z_{1}^{2}+z_{2}^{2}\right)+\varepsilon_{2} \\
& \leq-\lambda_{2} V+\varepsilon_{2}
\end{aligned}
$$

which leads to (34) and the results in (A) of Theorem 6 can be inferred, according to the deductive method in the proof of Theorem 4.

Now for part (B), when $d(x, t)=0$, from (27), similar to the derivation progress of part (A), the derivative of $V_{s}$ satisfies

$$
\dot{V}_{s} \leq-\sigma_{\min }\left(\Lambda_{2}\right)\left(z_{1}^{2}+z_{2}^{2}\right)+\widetilde{\theta}^{T}\left(\Gamma^{-1} \dot{\hat{\theta}}-\tau\right) .
$$

Noting the property P2 of (9), we have

$$
\dot{V}_{s} \leq-\sigma_{\min }\left(\Lambda_{2}\right)\left(z_{1}^{2}+z_{2}^{2}\right) .
$$

Therefore, similar to the proof of part (B) of Theorem 4 in Appendix A, the results in (B) of Theorem 6 can be proved, by applying Barbalat's lemma and the fact that all signals are bounded from part (A).

\section{Conflict of Interests}

The authors declare that there is no conflict of interests regarding the publication of this article.

\section{Acknowledgments}

The first author is grateful to Prof. B. Yao for hosting his guidance in ARC design. This work was supported in part by the National Natural Science Foundation of China under Grant 51305203 and Grant 51235002.

\section{References}

[1] M. Iwasaki, K. Seki, and Y. Maeda, "High-precision motion control techniques: a promising approach to improving motion performance," IEEE Industrial Electronics Magazine, vol. 6, no. 1, pp. 32-40, 2012.

[2] J. Huang, H. Li, Y. Chen, and Q. Xu, "Robust position control of PMSM using fractional-order sliding mode controller," Abstract and Applied Analysis, vol. 2012, Article ID 512703, 33 pages, 2012.

[3] A. Rincón, F. E. Hoyos, and F. Angulo, "Controller design for a second-order plant with uncertain parameters and disturbance: application to a DC motor," Abstract and Applied Analysis, vol. 2013, Article ID 169519, 16 pages, 2013.

[4] T. Shoji, S. Katsumata, S. Nakaura, and M. Sampei, "Throwing motion control of the springed pendubot," IEEE Transactions on Control Systems Technology, vol. 21, no. 3, pp. 950-957, 2013.

[5] B. Yao, C. Hu, L. Lu, and Q. Wang, "Adaptive robust precision motion control of a high-speed industrial gantry with cogging force compensations," IEEE Transactions on Control Systems Technology, vol. 19, no. 5, pp. 1149-1159, 2011.

[6] S. Lee and W.-J. Kim, "Active suspension control with directdrive tubular linear brushless permanent-magnet motor," IEEE Transactions on Control Systems Technology, vol. 18, no. 4, pp. 859-870, 2010.
[7] Y. Kim, P. LeDuc, and W. Messner, "Modeling and control of a nonlinear mechanism for high performance Microfluidic systems," IEEE Transactions on Control Systems Technology, vol. 21, no. 1, pp. 203-211, 2013.

[8] J. Yao, Z. Jiao, and D. Ma, "Adaptive robust control of DC motors with extended state observer," IEEE Transactions on Industrial Electronics, 2013.

[9] Y. Tan, J. Chang, and H. Tan, "Adaptive backstepping control and friction compensation for AC servo with inertia and load uncertainties," IEEE Transactions on Industrial Electronics, vol. 50, no. 5, pp. 944-952, 2003.

[10] R. H. A. Hensen, M. J. G. van de Molengraft, and M. Steinbuch, "Friction induced hunting limit cycles: a comparison between the LuGre and switch friction model," Automatica, vol. 39, no. 12, pp. 2131-2137, 2003.

[11] L. Xu and B. Yao, "Adaptive robust precision motion control of linear motors with negligible electrical dynamics: theory and experiments," IEEE/ASME Transactions on Mechatronics, vol. 6, no. 4, pp. 444-452, 2001.

[12] G. Tao and F. L. Lewis, Adaptive Control of Nonsmooth Dynamic Systems, Springer, New York, NY, USA, 2001.

[13] C. Makkar, W. E. Dixon, W. G. Sawyer, and G. Hu, "A new continuously differentiable friction model for control systems design," in Proceedings of the IEEE/ASME International Conference on Advanced Intelligent Mechatronics (AIM '05), pp. 600605, July 2005.

[14] J. S. Reed and P. A. Ioannou, "Instability analysis and robust adaptive control of robotic manipulators," IEEE Transactions on Robotics and Automation, vol. 5, no. 3, pp. 381-386, 1989.

[15] S. F. Alyaqout, P. Y. Papalambros, and A. G. Ulsoy, "Combined robust design and robust control of an electric DC motor," IEEE/ASME Transactions on Mechatronics, vol. 16, no. 3, pp. 574-582, 2011.

[16] Z. Z. Liu, F. L. Luo, and M. A. Rahman, "Robust and precision motion control system of linear-motor direct drive for highspeed X-Y table positioning mechanism," IEEE Transactions on Industrial Electronics, vol. 52, no. 5, pp. 1357-1363, 2005.

[17] S. I. Han and J. M. Lee, "Adaptive dynamic surface control with sliding mode control and RWNN for robust positioning of a linear motion stage," Mechatronics, vol. 22, no. 2, pp. 222-238, 2012.

[18] Y. Zhu and P. R. Pagilla, "Static and dynamic friction compensation in trajectory tracking control of robots," in Proceedings of the IEEE International Conference on Robotics adn Automation, pp. 2644-2649, May 2002.

[19] B. Armstrong-Hélouvry, P. Dupont, and C. C. De Wit, "A survey of models, analysis tools and compensation methods for the control of machines with friction," Automatica, vol. 30, no. 7, pp. 1083-1138, 1994.

[20] C. Canudas de Wit, H. Olsson, K. J. Åström, and P. Lischinsky, "A new model for control of systems with friction," IEEE Transactions on Automatic Control, vol. 40, no. 3, pp. 419-425, 1995.

[21] W.-F. Xie, "Sliding-mode-observer-based adaptive control for servo actuator with friction," IEEE Transactions on Industrial Electronics, vol. 54, no. 3, pp. 1517-1527, 2007.

[22] J. Yao, Z. Jiao, and B. Yao, "Robust control for static loading of electro-hydraulic load simulator with friction compensation," Chinese Journal of Aeronautics, vol. 25, no. 6, pp. 954-962, 2012.

[23] L. Márton and B. Lantos, "Control of mechanical systems with Stribeck friction and backlash," Systems \& Control Letters, vol. 58, no. 2, pp. 141-147, 2009. 
[24] L. Xu and B. Yao, "Output feedback adaptive robust precision motion control of linear motors," Automatica, vol. 37, no. 7, pp. 1029-1039, 2001.

[25] Y. Tan and I. Kanellakopoulos, "Adaptive nonlinear friction compensation with parametric uncertainties," in Proceedings of the American Control Conference (ACC '99), pp. 2511-2515, June 1999.

[26] L. Freidovich, A. Robertsson, A. Shiriaev, and R. Johansson, "LuGre-model-based friction compensation," IEEE Transactions on Control Systems Technology, vol. 18, no. 1, pp. 194-200, 2010.

[27] L. Lu, B. Yao, Q. Wang, and Z. Chen, "Adaptive robust control of linear motors with dynamic friction compensation using modified LuGre model," Automatica, vol. 45, no. 12, pp. 2890-2896, 2009.

[28] H. Armstrong and B. louvry, Control of Machines with Friction, Kluwer Academic Publishers, Boston, Mass, USA, 1991.

[29] B. Yao and M. Tomizuka, "Adaptive robust control of SISO nonlinear systems in a semi-strict feedback form," Automatica, vol. 33, no. 5, pp. 893-900, 1997.

[30] B. Yao, "Desired compensation adaptive robust control," Journal of Dynamic Systems, Measurement and Control, vol. 131, no. 6, article 61001, pp. 1-7, 2009.

[31] C. Makkar, G. Hu, W. G. Sawyer, and W. E. Dixon, "Lyapunovbased tracking control in the presence of uncertain nonlinear parameterizable friction," IEEE Transactions on Automatic Control, vol. 52, no. 10, pp. 1988-1994, 2007.

[32] J. Yao, Z. Jiao, and S. Han, "Friction compensation for low velocity control of hydraulic flight motion simulator: a simple adaptive robust approach," Chinese Journal of Aeronautics, vol. 26, no. 3, pp. 814-822, 2013.

[33] S. Sastry and M. Bodson, Adaptive Control: Stability, Convergence and Robustness, Prentice-Hall, Englewood Cliffs, NJ, USA, 1989.

[34] J. Yao, Z. Jiao, B. Yao, Y. Shang, and W. Dong, "Nonlinear adaptive robust control of electro-hydraulic load simulator," Chinese Journal of Aeronautics, vol. 25, no. 5, pp. 766-775, 2012.

[35] J. Yao, Z. Jiao, Y. Shang, and C. Huang, "Adaptive nonlinear optimal compensation control for electro-hydraulic load simulator," Chinese Journal of Aeronautics, vol. 23, no. 6, pp. 720-733, 2010.

[36] J. Yao, Z. Jiao, D. Ma, and L. Yan, "High accuracy tracking control of hydraulic rotary actuators with modelling uncertainties," IEEE/ASME Transactions on Mechatronics, 2013.

[37] O. G. Jepps, C. Bianca, and L. Rondoni, "Onset of diffusive behavior in confined transport systems," Chaos, vol. 18, no. 1, Article ID 013127, p. 13, 2008.

[38] M. Krstic, I. Kanellakopoulos, and P. V. Kokotovic, Nonlinear and Adaptive Control Design, John Wiley \& Sons, New York, NY, USA, 1995. 


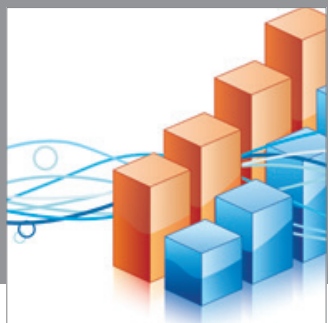

Advances in

Operations Research

mansans

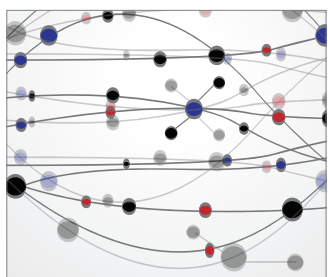

The Scientific World Journal
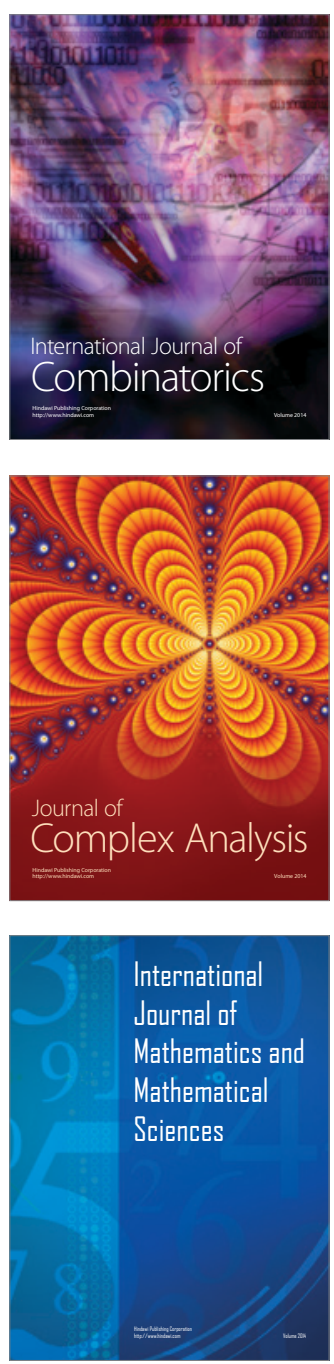
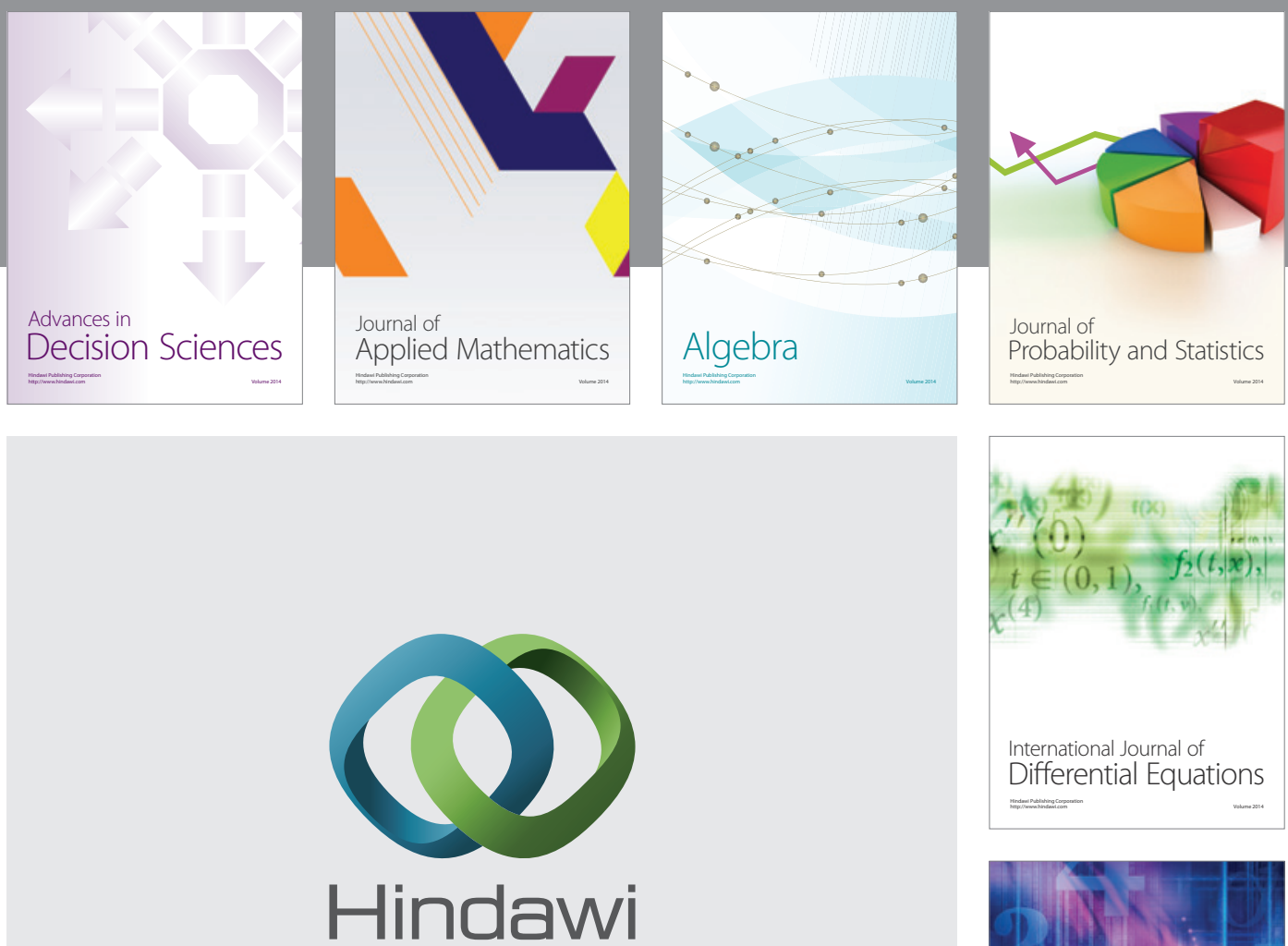

Submit your manuscripts at http://www.hindawi.com
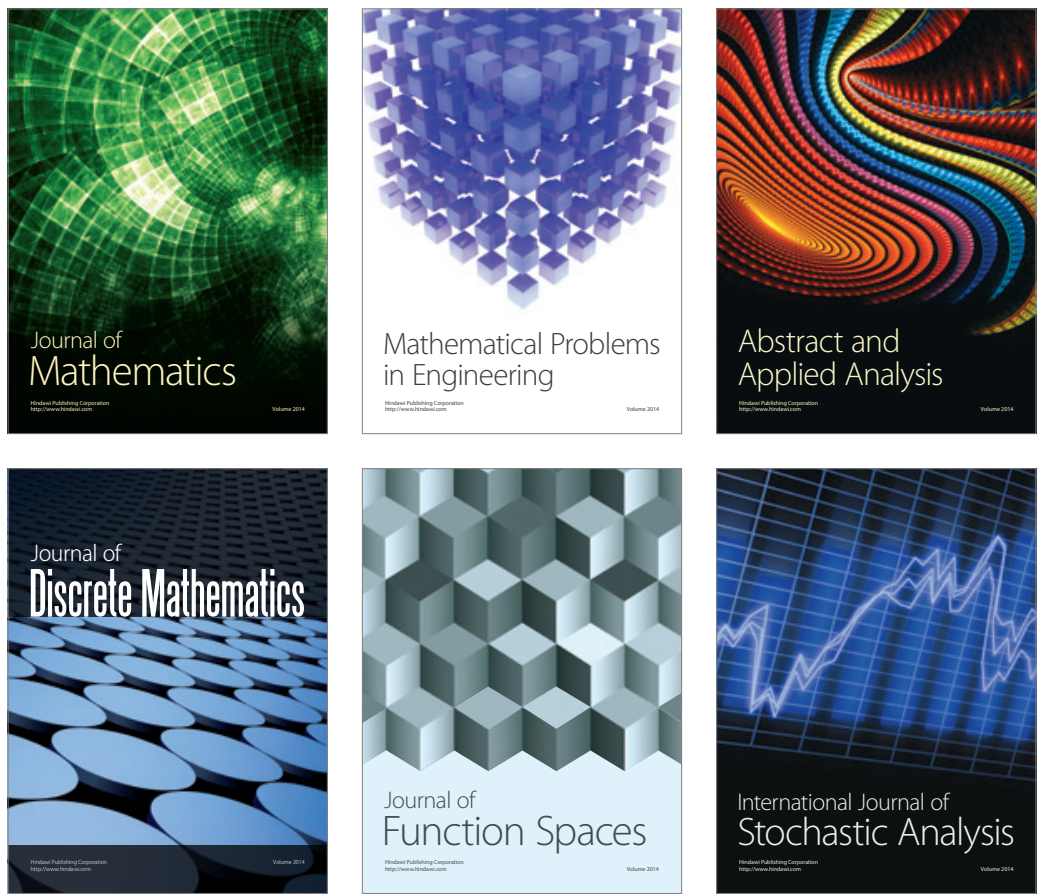

Journal of

Function Spaces

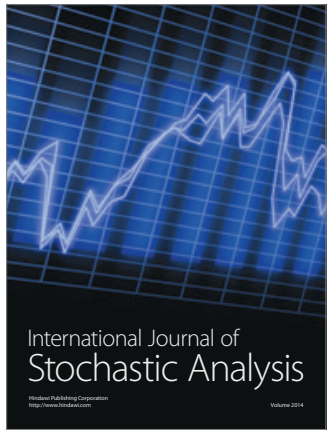

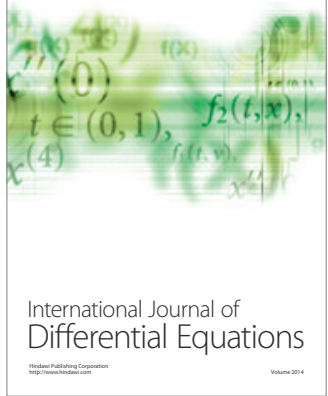
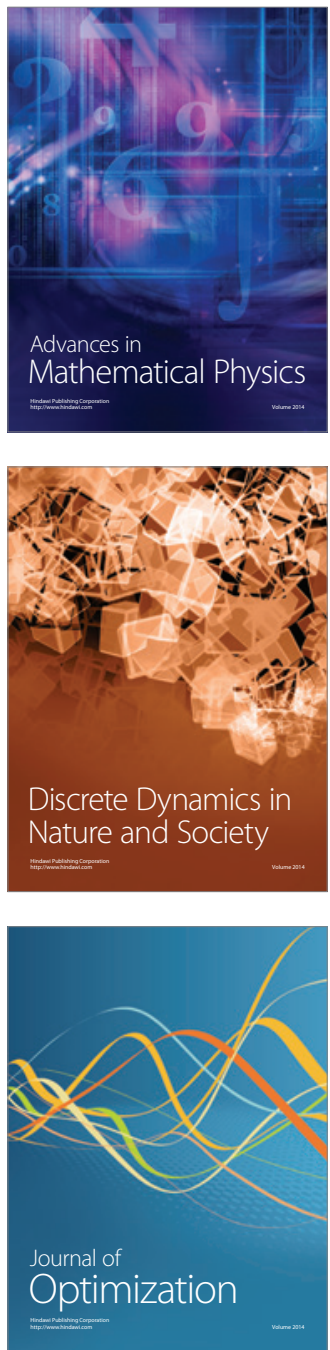